\title{
Statistical Structure and Mean Dynamics of Developing Turbulent Shear-Wake Flows
}

\author{
M. Bamberger · J. Klewicki
}

Received: date / Accepted: date

\begin{abstract}
Shear-wake flows formed from the interaction of two turbulent boundary layers are investigated. Primary focus is on the near-field downstream of the splitter plate. Two velocity ratios and two trailing edge geometries are explored via well-resolved hotwire traverses. Comparison with boundary layer data reveals that the loss of the no-slip condition is at first most apparent in the wall-normal velocity fluctuations. Estimates of the terms in the mean momentum equation are examined. Post-separation, the inertial terms in the mean momentum equation rapidly become dominant throughout the flow. Farther downstream the mean effect of turbulent inertia continues to change sign between the wake center and the freestream, as it does between the wall and freestream in the boundary layer. Unlike in the boundary layer, the mean and turbulent inertia terms retain leading order importance over the viscous force term everywhere.
\end{abstract}

Keywords Shear Wake - Turbulent Boundary Layer . Mixing Layer · Mean Momentum Balance

\footnotetext{
M. Bamberger

Department of Mechanical Engineering

University of New Hampshire

Durham, NH, 03824 E-mail: Marc.Bam@gmail.com

J. Klewicki

Department of Mechanical Engineering

University of New Hampshire

Durham, NH, 03824, USA

and

Department of Mechanical Engineering

University of Melbourne

Melbourne, Victoria, 3010, Australia

E-mail: joe.klewicki@unh.edu
}

\section{Introduction}

The present study investigates the flow field evolution arising from the interaction of two low Reynolds number turbulent boundary layers into a wake or shearwake flow, and, for the shear-wake, subsequently into a planar mixing layer flow. Turbulent boundary layer flows having an absolute velocity ratio of either 1:1 or 2:1 develop on opposite sides of a splitter plate as depicted in Fig. 1. At the end of the splitter plate these independently developed boundary layers begin to interact. The evolution of the flow structure within the initial post-separation zone is of primary interest, as this region lies between the boundary layer in-flow and mixing layer out-flow conditions. Clarity regarding features of this initial interaction is gained through comparisons between a sharp and blunt trailing edge geometry. Important elements effecting flow development include differences in velocity, opposing signs of mean vorticity, and the momentum deficit between the two layers caused by the plate thickness and no-slip condition. With downstream distance, the shear-wake flow evolves into a mixing layer, which has a single sign of mean vorticity (see Fig. 1), while the wake retains two signs of mean vorticity. For this and other reasons it is also useful to compare the evolution of the shear-wake with that of the symmetric wake.

A mechanistically descriptive and mathematically cogent framework for analyzing the present flow field evolution is described by Wei et al. (2005); Fife et al. (2005, 2009); Klewicki, Ebner \& Wu (2011). This framework is founded upon the dominant order balance of terms in the time-averaged statement of dynamics; revealing that the mean dynamics of the turbulent boundary layer are described by a four-layer structure. One attribute of this four layer structure is that the 
mean viscous force is of dominant order in the two layers closest to the wall. Conversely, existing data reveal that the mean momentum balance in the canonical freeshear flows (jets, wakes and mixing layers) is everywhere dominated by the terms that respectively account for inertia of the mean flow and the mean effect of turbulent inertia. All of these free-shear flows, however, have a wall-bounded origin. Given this, a primary aim is to clarify how the mean dynamics of wall-bounded flow evolves into that associated with free-shear turbulent flow.
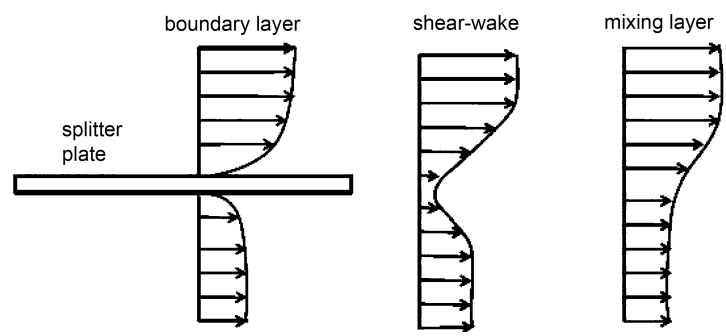

Fig. 1 Sketch of the mean streamwise velocity profile development from the boundary layer to mixing layer.

\subsection{Mean Flow Development}

While the boundary layer in-flow and two-stream mixing layer out-flow have been studied extensively, relatively few studies have explicitly focused on the downstream development of two dimensional shear-wakes that are formed from the interaction of turbulent boundary layers. Of these, perhaps the most significant is by Mehta (1991), who presented mean profile and turbulence statistics in the developing shear-wake for velocity ratios ranging between 0.5 and 1.0. This investigation, however, did not focus on the region just downstream of separation. Similarly, other studies that used the present facility explored shear-wake development for both laminar and turbulent in-flow boundary layers, see Wait (2003) and Challa (2005), respectively. The measurements of Challa (2005) are in good agreement with those presented herein. These previous experiments, however, only employed single element hotwire sensors, and thus did not quantify the behavior of the Reynolds shear stress and its cross-stream gradient.

Another related study is that by Morris \& Foss (2003), which quantified the evolution of a separating turbulent boundary layer into a single stream shear layer (effectively, a velocity ratio near zero). The most prominent feature revealed in this flow is the development of a "sub-shear layer," that forms from the wall-layer vorticity. On one side, the sub-shear layer interacts with the outer region of the separated boundary layer, and on the other side with the much slower entraining flow. This study also provided evidence that the boundary layer profile starts to distort near the wall almost immediately after separation; i.e., for $x / \theta>0.1$, where $\theta$ is the momentum deficit thickness of the boundary layer at separation. Important aspects of the developing shear-wake are also found in the inner shear layer region of developing co-axial jets, see, for example Ko \& Lam (1989); Dahm, Frieler \& Tryggvasan (1992); Sadr \& Klewicki (2003), and the references therein. The portion of this flow that is similar to shear-wake development has considerable technological significance, as this so-called intermediate zone is generally characterized by intense mixing. One of the present experiments includes finite plate thickness effects, as this parameter has been shown to influence flow behaviors in the intermediate zone.

There is also utility in viewing the shear-wake as the combination of a wake and mixing layer flow, as did Koochesfahani and Frieler (1989). Such a perspective naturally leads one to consider the properties of the decay of the wake component. That is, even though it is clearly recognized that the turbulent shear-wake is not a linear superposition of a wake and mixing layer, insights are gained by comparing with the turbulent near-wake of a flat plate. Experimental studies of this flow include those by Chevray \& Kovasznay (1969), Andreopoulos \& Bradshaw (1980), Haji-Haidari \& Smith (1988), and Nakayama \& Liu (1990), while theoretical/analytical treatments include those by Alber (1980) and Bogucz \& Walker (1988). Relative to mean profile development, the analysis of Haji-Haidari \& Smith (1988) includes the data of Chevray \& Kovasznay (1969) and Andreopoulos \& Bradshaw (1980). They show that, to a good approximation, the wake centerline velocity follows a logarithmic variation in the streamwise direction $\left(x^{+}\right)$that is akin to the logarithmic variation in wall-normal direction $\left(y^{+}\right)$in the turbulent boundary layer. Nakayama \& Liu (1990) give evidence that the logarithmic variation always exists, but that the logarithmic curve shifts upward with increasing boundary layer Reynolds number. They attribute this effect to variations in the influence of the large scale motions in the boundary layer with increasing Reynolds number. Bogucz \& Walker (1988) provide an analysis based upon a two-layer structure and matched expansions that, independent of turbulence model, apparently captures the downstream scaling behavior of the mean velocity and Reynolds stress in the near-wake. One element of the present effort is to explore whether features of the wake development persist in the presence of a mean shear. 
The evolution from the boundary layer to the mixing layer is illustrated in Fig. 1. The abrupt end of the splitter plate marks the loss of the no-slip boundary condition, and thus also marks the initial center of the developing wake component of the shear-wake flow. With the loss of the solid boundary, the inner regions of the two separated boundary layers may exchange momentum. Finite plate thickness effects depend on the trailing edge geometry. If the plate has a sharp trailing edge, the boundary layers will interact immediately. If the trailing edge is blunt, the separated boundary layers will develop approximately independently for some streamwise fetch prior to interacting. The flow behaviors associated with these phenomena are thus of interest. The mean profile downstream of separation evolves (at different rates depending of the geometry of the trailing edge) to one having a curvature that is distinct from either the boundary layer or the mixing layer. This wake component diminishes with downstream development as the bounding shear layers supply it with momentum.

The boundary layer Reynolds stress profile is zero at the wall and rises to a peak magnitude that is near unity at a $y^{+}\left(=y u_{\tau} / \nu\right)$ position that varies like the square root of the Reynolds number, e.g., Long \& Chen (1981); Afzal (1982); Sreenivasan \& Sahay (1997); Wei et al. (2005). Note that boundary layer Reynolds number is herein denoted by $\delta^{+}=\delta u_{\tau} / \nu$, where $\delta$ is the boundary layer thickness, $u_{\tau}=\sqrt{\tau_{w} / \rho}$ is the friction velocity and $\nu, \tau_{w}$, and $\rho$ are the kinematic viscosity, mean wall shear stress, and mass density respectively. Mixing in the shear-wake is generally intense, as the inner regions of the boundary layers interact to create large turbulent fluctuations. As shown herein, these fluctuations correlate to generate a rapid increase in the peaks of the Reynolds stress. Farther downstream, these peaks decay until there is a single Reynolds stress peak in the mixing layer. The position of this maximum is offset towards the high velocity side depending on the value of the velocity ratio, Mehta (1991).

The magnitude of the mean vorticity profile, $\Omega_{z} \simeq$ $-d U / d y$, in the boundary layer is maximal at the wall, and decreases with increasing distance from the wall - at first rapidly, and then much more gradually. It is worth reiterating that the mean vorticity profiles in the pre-separation boundary layers are of opposing sign, and that the total circulation per unit length in each boundary layer has a magnitude equal to the free stream velocity. These characteristics underlie important phenomena that are initiated when the plate ends and the vorticity fields in the two streams interact. Namely, the momentum transport leading to the reduction of the wake component coincides with the annihi- lation of the opposing sign mean vorticity of the in-flow boundary layers. This cancelling of vorticity continues downstream until the larger magnitude $\Omega_{z}$ in the higher velocity flow eventually dominates. During this process, the peaks in $\Omega_{z}$ no longer reside at the $y$ location of the wall.

\subsection{Properties of the Mean Momentum Balance}

The mean dynamical equation for the zero pressure gradient turbulent boundary layer is given by

$\rho\left(U \frac{\partial U}{\partial x}+V \frac{\partial U}{\partial y}+\frac{\partial\langle u v\rangle}{\partial y}+\frac{\partial\left(\left\langle u^{2}\right\rangle-\left\langle v^{2}\right\rangle\right)}{\partial x}\right)=\mu \frac{\partial^{2} U}{\partial y^{2}}$,

where an upper case denotes a mean quantity, lower case denotes a fluctuation about the mean, and angle brackets denote time averaging. On the left of (1) the first two terms represent the inertia of the mean flow $(M I)$, while the third term describes the time averaged effect of turbulent inertia $(T I=-\rho \partial\langle u v\rangle / \partial y)$. The fourth term on the left (streamwise gradient term) is also a mean effect of turbulent inertia, but is of negligible magnitude in the zero pressure gradient boundary layer. This term is included in (1) since its effects will be considered in the developing shear-wake. The terms on the left are balanced by the mean viscous force $(V F)$ on the right. While (1) generically holds, not all of its terms are of leading order throughout the entire boundary layer. This is distinct from the terms in the laminar boundary layer equation.

Wei et al. (2005) were the first to reveal that the magnitude ordering of the terms in (1) is described by the four layer structure depicted in Fig. 2. In layer I the primary balance is between $M I$ and $V F$, although the details of this depend on the rates at which all the terms approach zero as $y^{+} \rightarrow 0$, see Wei et al. (2007); Klewicki, Ebner \& Wu (2011). In layer II the leading order balance is between $V F$ and $T I$. The layer II ratio is increasingly well-approximated by -1 as $\delta^{+}$becomes large. Similarly, in layer IV the leading order balance is between $M I$ and $T I$, and thus $V F / T I \simeq 0$. Owing to the exchange of balance from layer II to layer IV, the $M I, T I$ and $V F$ terms of (1) have leading order importance in layer III. Thus, the mean dynamics from layer II to layer IV are characterized by a balance breaking and exchange of terms that is directly coupled to the fact that $T I$ changes sign - or equivalently, $|\langle u v\rangle|$ passes through its maximum, e.g., Fife et al. (2005, 2009). As shown herein, the $|\langle u v\rangle|$ peaks affiliated with the individual boundary layers are retained for small $x / \theta$ in the shear-wake. This has implications regarding how the balance of terms in (1) is realized in the shear-wake. 
Examination of existing data (including those herein) reveals that the leading order mean dynamics in the mixing layer are described by a balance between the $M I$ and $T I$ terms in (1). Distinct from the relatively complex layer structure depicted in Fig. 2, the ratio of $V F$ to $T I$ in the mixing layer is therefore approximately zero everywhere. Within the flow domain of the shearwake the mean momentum balance changes from that of Fig. 2, to one described by a single horizontal line at $V F / T I \simeq 0$ everywhere (not shown). An important aim of the present study is to clarify how this occurs.

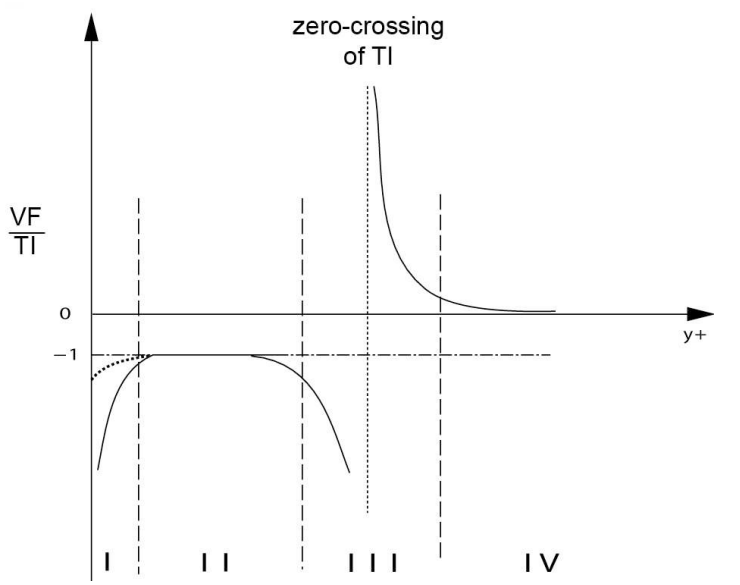

Fig. 2 Sketch of the ratio of the mean viscous force to the mean effect of turbulent inertia in turbulent wall-flows. The profile of this ratio graphically reveals the magnitude ordering of the terms in (1) as a function of distance from the wall. Note that the behavior of layer I in the boundary layer (dotted line in layer I) is different from that in the pipe or channel, e.g., see Wei et al. (2005, 2007); Klewicki, Ebner \& $\mathrm{Wu}$ (2011).

\section{Experiment}

\subsection{Two-Stream Shear Layer Facility}

The experiments were conducted in a two-stream shear layer wind tunnel, a schematic of which is shown in Fig. 3. The flow is driven by two centrifugal blowers each controlled by a separate variable frequency drive. The tunnel velocity range is between about $2 \mathrm{~m} / \mathrm{s}$ and $20 \mathrm{~m} / \mathrm{s}$. Downstream of the centrifugal blowers, each stream passes through a honeycomb and series of screens prior to accelerating through a $3: 1$ contraction, and into the approximately $4 \mathrm{~m}$ long test section. A strip of $0.076 \mathrm{~m}$ wide 50 grit sand paper glued to the splitter plate at the test section inlet is used to trip the boundary layer to turbulence about $1.2 \mathrm{~m}$ upstream of the end of plate. The height of the test section is $0.91 \mathrm{~m}$, and the shear-wake measurements were acquired near the vertical centerline.

The test section consists of a double-wall construction. There is an outer fixed wall (not shown in Fig. 3), and an inner false wall that can be adjusted to set the axial pressure gradient. This adjustable wall is hinged at the beginning of the test section. Prior to the present experiments, the inner walls were iteratively adjusted to establish a nominally zero pressure gradient flow. The required wall divergence angle was found experimentally via streamwise Pitot-static tube surveys in the test section. The wall divergence angle was ultimately set at about 0.53 degrees. During the experiments the free-stream velocities on each side of the splitter plate were monitored using two Pitot-static tubes mounted through the top of the tunnel near the inlet to the test section.

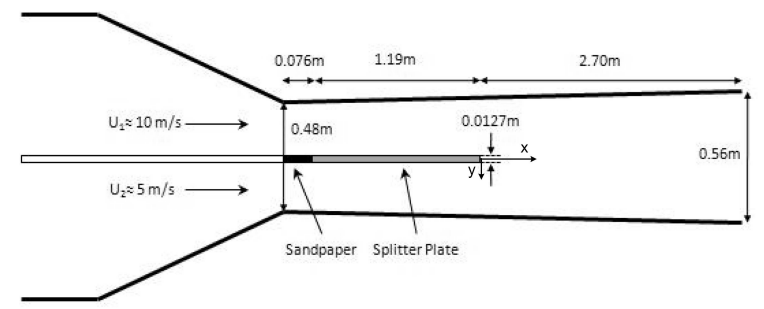

Fig. 3 Dimensioned schematic of the experimental facility.

The splitter plate had a baseline thickness of $1.27 \mathrm{~cm}$, and from this two different trailing edge geometries were configured. For the blunt trailing edge the splitter plate simply remained constant thickness; abruptly ending at a 90 degree corner. For the sharp trailing edge, the splitter plate was linearly and symmetrically tapered over a streamwise distance of $12.6 \mathrm{~cm}$, resulting in a wall divergence of about 2.9 degrees. Thus, for the sharp edge experiments, the boundary layers experienced a short length of adverse pressure gradient just prior to separation. Evidence of this effect is given later.

\subsection{Hot-wire Experiments}

The data presented herein are derived from measurements using an in-house built x-array hotwire sensor. The wires on the sensor are $2.5 \mu \mathrm{m}$ diameter Wollaston wire with a platinum $/ 10 \%$ rhodium core. The support prongs are constructed out of $304 \mathrm{~V}$ stainless steel. The prongs were tapered (via a specially designed grinding system) over a distance of about $1 \mathrm{~cm}$ with a final tip diameter of about $35 \mu \mathrm{m}$. The prongs have a spacing of $0.44 \mathrm{~mm}$. The x-array wires are nominally oriented at 45 
degrees to the probe axis, which yields a wire length of about $0.62 \mathrm{~mm}$. Under the worst case (i.e., in the $10 \mathrm{~m} / \mathrm{s}$ flow), $0.62 \mathrm{~mm}$ corresponds to about 18.8 viscous units. Experiments in low $\delta^{+}$boundary layers indicate that probes of this non-dimensional size will have between $5 \%$ and $10 \%$ signal attenuation resulting from spatial averaging, e.g., Klewicki \& Falco (1990). The wire resistance is about $30 \Omega / \mathrm{mm}$. The sensors were operated using AA Lab Systems AN 1003 constant temperature anemometers that were adjusted to accommodate the resistance of the present sensor. The wires on the sensor were operated at a heating ratio of 1.3 , and their impulse frequency response was estimated to be greater than $30 \mathrm{kHz}$. This is nearly three times the highest frequency measured in the flow, and over twice the frequency at which the data were sampled.

The sensor was calibrated using an articulating jet that incorporated elements of the design previously used by Folz \& Wallace (2009). Velocities in the jet were determined via the pressure difference across the nozzle. These pressure differences were measured using an MKS 69810 Torr transducer. The angle sweep of the calibration was \pm 30 degrees. The present calibration methodology employs Jorgensen's equation as implemented in Vukoslavcevic, Wallace \& Balint (1991); Vukoslavcevic \& Wallace (1996).

Measurements were acquired in three flow fields: $(i)$ a $U_{\infty}=10 \mathrm{~m} / \mathrm{s}$ symmetric wake downstream of the sharp trailing edge, (ii) a shear-wake downstream of the sharp edge with high and low speed streams of $5 \mathrm{~m} / \mathrm{s}$ and $10 \mathrm{~m} / \mathrm{s}$ and,(iii) a shear-wake of the same free stream flow speeds, but developing downstream of the blunt trailing edge. Profile measurements were acquired at up to eight streamwise locations downstream of the separation edge. These positions are given dimensionally in Table 1 , as normalized by $\nu$ and $u_{\tau}$, and as normalized by $\theta$, the momentum deficit thickness of the first profile downstream of separation. For all of the experiments the data were sampled at $12 \mathrm{kHz}$. Data were sampled for 40 s at each location. For the worst case, i.e., in the $5 \mathrm{~m} / \mathrm{s}$ boundary layer, this corresponds to the passage of about 10,000 $\delta$-scale eddies, and thus should yield statistical convergence for the quantities presented to well within 1\%, e.g. Klewicki \& Falco (1990).

The velocity and Reynolds stress data presented herein are primarily normalized by the estimate for the friction velocity at separation. This estimate was found using the Clauser plot method and the mean profile at $x / \theta=1.76$, which is the first measurement location downstream of separation. Although this estimate of $u_{\tau}$ is relatively crude, this normalization still proves useful for revealing the rapid changes in magnitude that occur in the turbulent stresses downstream of separa-

\begin{tabular}{ccc|ccc}
\hline \multicolumn{3}{c|}{ Blunt Edge } & \multicolumn{3}{c}{ Sharp Edge } \\
$(x / \theta)$ & $x^{+}$ & $(\mathrm{mm})$ & $(x / \theta)$ & $x^{+}$ & $(\mathrm{mm})$ \\
\hline \hline 1.76 & 116 & 3.80 & 1.76 & 116 & 3.80 \\
2.94 & 194 & 6.35 & 2.94 & 194 & 6.35 \\
5.88 & 387 & 12.70 & 5.88 & 387 & 12.70 \\
8.82 & 580 & 19.05 & 8.82 & 580 & 19.05 \\
11.76 & 774 & 25.40 & 11.76 & 774 & 25.40 \\
41.16 & 2709 & 88.91 & 41.16 & 2709 & 88.91 \\
82.31 & 5417 & 177.79 & 99.95 & 6578 & 215.89 \\
158.75 & 10447 & 342.90 & - & - & \\
\hline
\end{tabular}

Table 1 Downstream profile locations for blunt and sharp trailing edge geometries.

tion. For purposes of comparing with the properties of the boundary layer, it is sometimes useful to normalize $y$ or $x$ by $\nu$ and $u_{\tau}$ (i.e., $y^{+}=y u_{\tau} / \nu$ ) from the high speed side. This is generally restricted to the region $x / \theta<10$. Otherwise we employ the shear layer thickness, $\delta_{s w}(x)$, which is defined as the distance between the positions where the mean velocity attains $99 \%$ of the respective free stream velocity on each side of the shear-wake. The scaled cross-stream coordinate, $\eta$, thus becomes $\eta=y / \delta_{s w}(x)$. Additional details pertaining to the experiments may be found in Bamberger (2011).

\section{Results}

\subsection{Turbulent Boundary Layer Comparison}

Prior to a detailed exploration of the shear-wake development, it is instructive to compare the mean flow properties at the first measurement station downstream of the separation edge with those of a canonical turbulent boundary layer at comparable Reynolds number. The $10 \mathrm{~m} / \mathrm{s}$ stream is common to all of the experiments. Thus, one can think of the present experiments as documenting the evolution of a separated $\delta^{+} \simeq 570(10 \mathrm{~m} / \mathrm{s})$ boundary layer subjected to a spatially varying boundary condition on its low speed side. These variations are dictated by parameters such as the velocity ratio of the two streams, and the effects caused by the geometry of the trailing edge. Thus, in what follows we compare properties of the separated $10 \mathrm{~m} / \mathrm{s}$ boundary layer at $x / \theta=1.76$ to quantities derived from the recent direct numerical simulation (DNS) of Schlatter \& Orlu (2010).

Parameters associated with the separated boundary layers at $x / \theta=1.76$ are given in Table 2 . The low Reynolds number of these flows has implications that are discussed in the data presentation. Relative to the flow field evolution away from the four-layer structure discussed in $\S 1$, it is important to note that the recent estimates of Klewicki, Ebner \& Wu (2011) put the on- 
Table 2 Properties of the Separated Boundary Layers at $x / \theta=1.76$

\begin{tabular}{ccccccccc}
\hline $\begin{array}{c}\text { Tunnel } \\
\text { Side }\end{array}$ & $\begin{array}{c}U_{\infty} \\
(\mathrm{m} / \mathrm{s})\end{array}$ & $\begin{array}{c}u_{\tau} \\
(\mathrm{m} / \mathrm{s})\end{array}$ & $\begin{array}{c}\delta_{99} \\
(\mathrm{~mm})\end{array}$ & $\begin{array}{c}\delta^{*} \\
(\mathrm{~mm})\end{array}$ & $\begin{array}{c}\theta \\
(\mathrm{mm})\end{array}$ & $\delta^{+}$ & $R_{\theta}$ & $\mathrm{H}$ \\
\hline \hline Low Speed & $\simeq 5$ & $\simeq 0.24$ & 20.4 & 2.0 & 1.7 & 320 & 580 & 1.20 \\
High Speed & $\simeq 10$ & $\simeq 0.46$ & 18.7 & 2.8 & 2.2 & 570 & 1390 & 1.31 \\
\hline
\end{tabular}
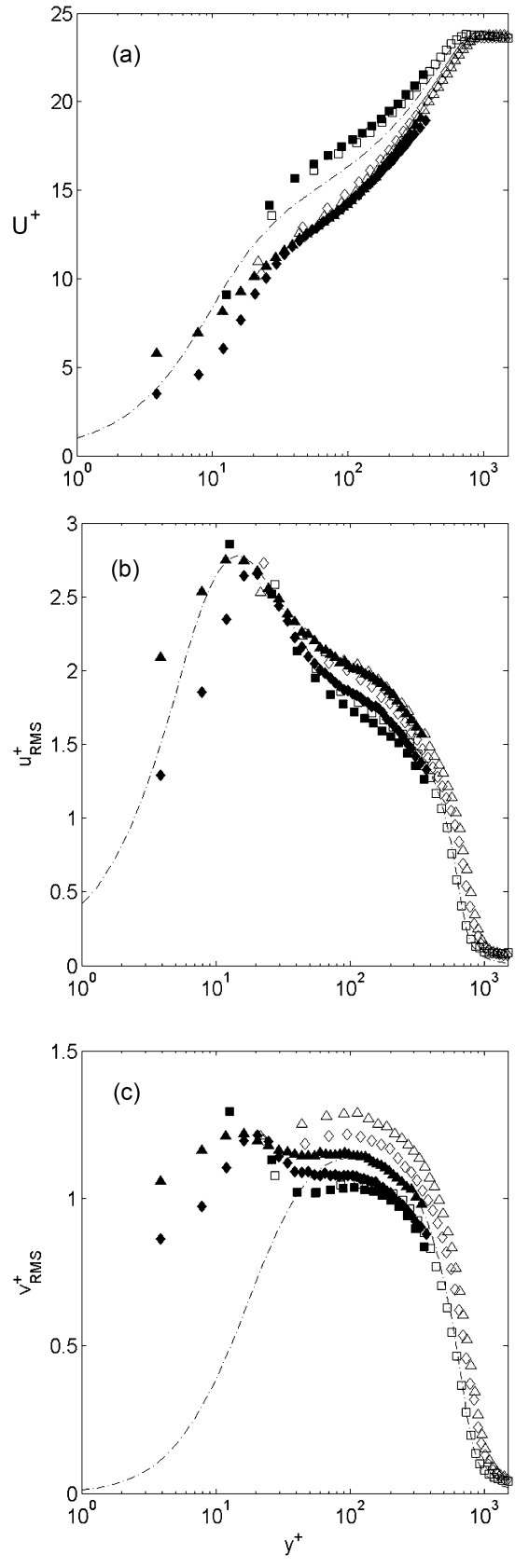

Fig. 4 Inner-normalized (a) mean velocity profiles, (b) streamwise velocity intensities, and (c) wall-normal velocity intensities from the separated $\delta^{+}=570$ boundary layers at $x / \theta=1.76$. Blunt trailing edge shear-wake: squares, sharp trailing edge shear-wake: diamonds, and the sharp trailing edge wake: triangles. Solid symbols denote higher data density near-wall profiles. Dot-dash profiles: DNS of Schlatter \& Orlu $(2010)$ at $\delta^{+} \simeq 670$. set of the four layer regime at $\delta^{+} \simeq 360$. Thus, the high speed flow has just attained the magnitude ordering of terms and scaling properties characteristic of the four layer regime, while the low speed has not quite entered this regime.

Figure $4 \mathrm{a}$ presents a comparison of the mean velocity profiles. Note that to facilitate comparisons, in these profiles $y^{+}=0$ in the blunt trailing edge corresponds with the plane of the plate, not the center of the plate. Note also that for the purposes of obtaining more accurate derivatives, near-wall profiles were replicated at nominally the same Reynolds number but with more closely spaced data. These profiles are given by the solid symbols on the figure. In this and subsequent plots that compare the three flows, square symbols denote the blunt trailing edge shear-wake, diamonds denote the sharp trailing edge shear-wake, and triangles denote the sharp trailing edge wake. As indicated by a number of plots herein, the data from the sharp trailing edge configuration (both with and without shear) exhibit a greater degree of distortion from the canonical case than the comparable data from the blunt trailing edge configuration with shear. The primary reason for this is believed to be associated with the short segment of adverse pressure gradient caused the taper of the splitter plate. After separation from the sharp trailing edge, the two boundary layers immediately undergo a direct interaction. Conversely, the post-separation blunt trailing edge flow is at first akin to the single stream separation problem studied by Morris \& Foss (2003). Their mean profile measurements provide evidence of a near-wall distortion that is small, but detectable, for $x / \theta \geq 0.1$.

The comparisons between the present streamwise and wall-normal velocity intensities and the corresponding DNS profiles are given in Figs. 4b,c respectively. Interior to $y^{+}=30$ the $v^{\prime+}$ profiles are clearly distorted, while the near-wall $u^{\prime+}$ profile distortions are much less apparent. For greater $y^{+}$, the streamwise intensity profile from the blunt trailing edge configuration exhibits good agreement with the DNS profile, while the wallnormal intensity profile has a similar shape, but slightly smaller peak amplitude. Some of this difference is rationally attributable to the higher Reynolds number of the DNS, as the amplitude of the wall-normal intensity profile is known to increase significantly with Reynolds number at low $\delta^{+}$. The sharp trailing edge 
profiles exhibit behaviors that may be associated with the aforementioned fetch of adverse pressure gradient just prior to separation. Outside of the near-wall region the streamwise intensity profiles attain elevated values relative to the canonical case, while high and low density wall-normal intensity profiles fall below and above the DNS profile, respectively. It is presently unknown how much of the apparent difference between the sharp trailing edge intensity profiles can be attributed to the different velocity of the adjacent stream versus the uncertainty of the $u_{\tau}$ estimate.

\subsection{Evolution of the Mean Streamwise Velocity}

In this and the following subsections we describe elements of the flow field evolution by presenting sequences of the profile data at the $x / \theta$ positions indicated in Table 1. Owing to the nature of the flow development, the presentation of these profiles is generally divided into two approximate regions, $x<10 \theta$ and $x>10 \theta$. In each subsection, the blunt trailing edge shear-wake profiles are presented first, followed by those for the sharp trailing edge shear-wake, and then those for the sharp trailing edge wake.

Figure 5a shows the evolution of the blunt trailing edge $U^{+}$profiles as a function of $\eta=y / \delta_{s w}$ for the indicated $x / \theta$ range. In this and subsequent figures, the vertical dot-dash lines indicate the planes of the splitter plate surfaces. For small $x / \theta$ the accuracy of the measurements interior to these lines is diminished owing to the low velocities, and, at the smallest $x / \theta$, the likelihood of high flow angles. It is apparent that during the initial development of the blunt trailing edge shearwake the two boundary layers do not directly interact. Instead, the near-wall vorticity field on each side interacts with the low velocity flow immediately behind the plate. This is similar to the initial flow development for the single stream shear layer study of Morris $\&$ Foss (2003), although the entrainment flow in the present case is dictated by the bounding shear layers rather than being externally controlled. For $x / \theta<10$, the data on both sides of the splitter plate respectively merge onto a single curve, while farther downstream these profiles diverge in the outer region. This is consistent with the observations of Morris \& Foss (2003) that the outer flow is at first unaffected by the removal of the no-slip boundary condition. The profiles of Fig. 5 provide evidence that somewhere between $x / \theta=8.82$ and $x / \theta=11.76$ the wall-layer vorticity fields of the separated boundary layers begin to directly interact. Thereafter, the profile development is marked by a monotonic migration of the minimum wake component of the profile toward the low speed side. At the farthest down- stream measurement location $(x / \theta=158.8)$ there remains a small but discernible wake component.

In contrast, the $U^{+}$profiles of Fig. $5 \mathrm{~b}$ provide evidence that in the sharp trailing edge shear-wake direct interactions between the separated boundary layers are immediately significant. For example, by $x / \theta=1.76$ the minimum mean velocity has increased from its value of 0 (in the presence of a wall boundary condition) to about $15 \%$ of the high speed stream. Outside of this relatively narrow region where the two streams interact, the initial boundary layer profiles remain largely unchanged. This is taken to indicate that the initial interactions are at first spatially localized, and then spread laterally. Relative to observations in both the single stream shear layer and the symmetric wake (e.g., Morris \& Foss (2003); Andreopoulos \& Bradshaw (1980); Haji-Haidari \& Smith (1988)), this initial rate of lateral spreading is likely to be associated with interactions primarily involving vorticity originating from the viscous wall layers of the parent boundary layers. Close examination of the profiles for $x / \theta<10$ indicates that the position of minimum wake component almost immediately begins to migrate toward the low speed side. Furthermore, comparison with Fig. 5a for $x / \theta>10$ reveals that this phenomenon is highly similar to that found in the blunt trailing edge experiment. These behaviors are explicitly quantified in Fig. 8 below.

It is rational to hypothesize that the intensity of the interactions effecting momentum transport between the two streams is strongly coupled to the mean vorticity amplitude of the two streams. Somewhat surprisingly, comparison of the shear-wake mean profiles (velocity ratio $=2$ ) of Fig. $5 \mathrm{~b}$ to the wake profiles (velocity ratio $=1$ ) reveals that the minimum measured wake velocities at $x / \theta=1.76$ are nearly the same. From a comparison of the wake deficit at the furthest downstream location it is also tempting to surmise that the rate at which the wake compenent gains momentum with downstream development is noticeably slower than in either of the shear-wake cases. The wake and shearwake flows, however, have qualitatively different endstates. Thus, while the profiles of Figs. 5a, b are closer their mixing layer end-state at comparable $x / \theta$, the absolute change (increase) in the wake minimum is largest for the symmetric wake.

$U_{m}$ denotes the value of the mean velocity where the wake component of the shear-wake profile is maximal. For the symmetric wake this is the mean velocity at the wake centerline. Properties relating to $U_{m}$ are quantified in Figs. 6 - 8. Previous experimental and analytical studies of the near-wake downstream of a sharp trailing edge indicate that for a range of $x^{+}>300$ the inner-normalized wake centerline velocity exhibits 

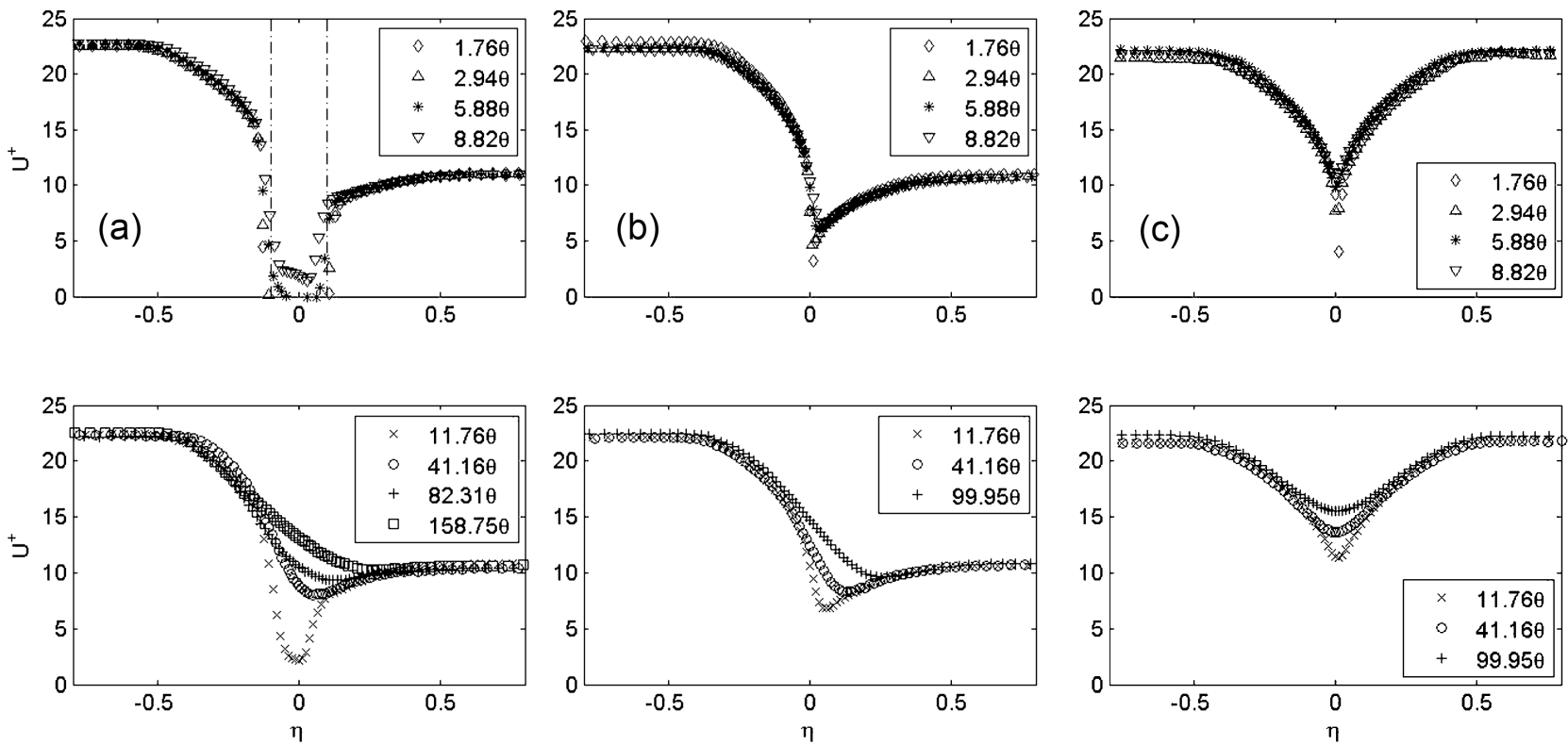

Fig. 5 Mean velocity profile evolution: (a) shear-wake flow downstream of the blunt trailing edge (dot-dash lines indicate the $\eta$ positions of the splitter plate surface), (b) shear-wake flow downstream of the sharp trailing edge, (c) wake flow downstream of the sharp trailing edge. Note that $\eta=y / \delta_{s w}$ and that entries in the legend denote streamwise location downstream of the splitter plate.

a logarithmic-like dependence (Andreopoulos \& Bradshaw (1980); Haji-Haidari \& Smith (1988); Nakayama \& Liu (1990)). The wake data of Fig. 6 further support these findings. A logarithmic curve-fit of the present wake data yields a slope of about 1.92. This is slightly less than the value of 2.12 found by Andreopoulos \& Bradshaw (1980), but significantly less than the value of about 2.45 found by Haji-Haidari \& Smith (1988). As noted previously, the trailing edge taper of the present experiments is 2.9 degrees. This compares to about $1.2,1.8$, and 4 degrees in the studies by Nakayama \& Liu (1990), Andreopoulos \& Bradshaw (1980), and Haji-Haidari \& Smith (1988), respectively.

The sharp trailing edge shear-wake data of Fig. 6 also exhibit a logarithmic-like dependence, and thus the presence of a non-zero circulation for the flow as a whole does not destroy this feature of the wake component development. When normalized by the $u_{\tau}$ value from the low speed boundary layer the slope of the logarithmic region in this profile is about 2.45 , but when normalized by $u_{\tau}$ from the high speed side the slope is about 1.25. Remarkably, the present blunt trailing edge data provide evidence that after the separated shear layers spread across the plate thickness (i.e., for $x / \theta>10$ ), $U_{m}$ undergoes a downstream development similar to that in the sharp edge shear-wake. As in the case of the study of Haji-Haidari \& Smith (1988), the present estimates for $u_{\tau}$ in the sharp trailing edge flows do not include the effect of the tapered portion preceding sep-

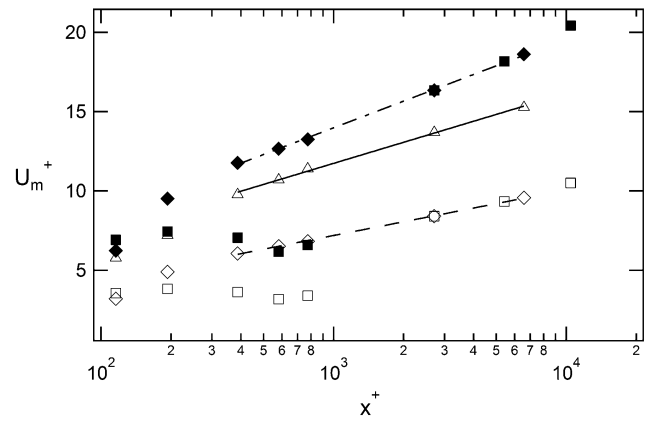

Fig. 6 Downstream evolution of $U_{m}$ as a function of $x^{+}$. Symbols same as in Fig. 4. Solid line, $U_{m}^{+}=1.92 \ln \left(x^{+}\right)-1.5$, dashed line, $U_{m}^{+}=1.25 \ln \left(x^{+}\right)-1.44$, dot-dash line, $U_{m}^{+}=$ $2.43 \ln \left(x^{+}\right)-2.80$. Open symbols normalized by $u_{\tau}$ from the high speed boundary layer, solid symbols normalized by $u_{\tau}$ from the low speed boundary layer.

aration. This factor influences the observed value of the logarithmic slope in Fig. 6.

Figure 7 displays the ratio $U_{m} / U_{\infty}\left(U_{\infty} \simeq 10 \mathrm{~ms}\right)$ versus $x / \theta$ on logarithmic axes for all of the present flows. Similar to the data of Fig. 6, the sharp trailing edge shear-wake data approximately fall along a power law fit that has a slightly larger slope than for the wake. Prior to the direct interaction between the separated boundary layers in the blunt trailing edge flow, this ratio exhibits no discernible trend. (Part of this may be affiliated with the veracity of the measurements directly behind the plate.) For $x / \theta>10$ (see Fig. 5), the dependence of $U_{m} / U_{\infty}$ on $x / \theta$ is very similar to that 


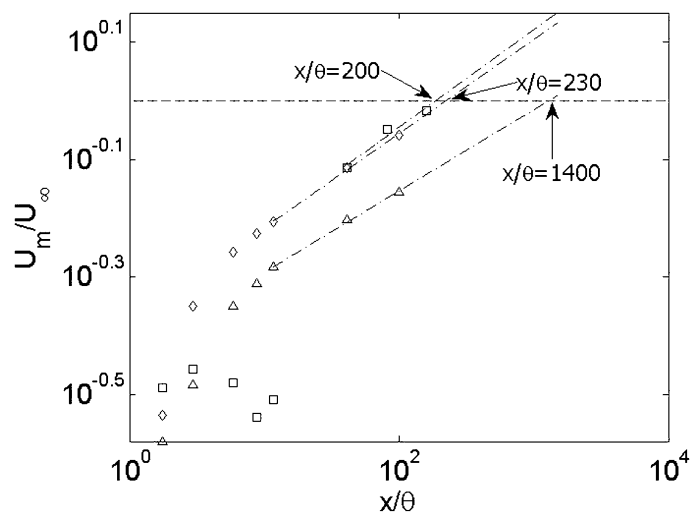

Fig. 7 Evolution of $U_{m} / U_{\infty}$ as a function $x / \theta$. Symbols same as in Fig. 4.

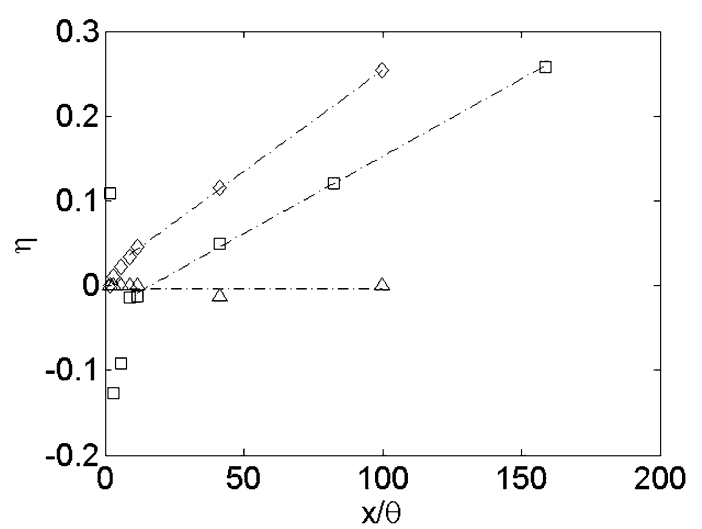

Fig. 8 Center position of the profile wake-component as a function $x / \theta$. Symbols same as in Fig. 4 .

for the sharp trailing edge shear-wake. As graphically depicted in Fig. 7, the power law fits allow extrapolation to the point where the wake component disappears. These rough estimates are given by $x / \theta=200,230$, and 1400 for the blunt trailing edge shear-wake, sharp trailing edge shear-wake and wake flows, respectively.

In the case of the symmetric wake, the position of $U_{m}$ remains fixed along the axis of the splitter plate. As indicated by the data of Fig. 5, in the shear-wake the position of the wake component center continuously shifts toward the low-speed side with increasing $x / \theta$. The data of Fig. 8 indicate that for $x / \theta>10$ the shift in this $\eta$ position exhibits a linear dependence on $x / \theta$ in both of the shear-wake flows. For $x / \theta<10$, the blunt trailing edge data are scattered, but by $x / \theta=5.88$ they begin to locate near $\eta=0$. The sharp edge shear-wake data for $x / \theta<10$ exhibit an approximately linear shift upward to a position near $\eta=0.05$.

\subsection{Turbulence Intensities and Reynolds Stresses}

For $\delta^{+}$greater than about 360, the mean structure of boundary layer includes a region (extending to $y^{+} \simeq$ $2.6 \sqrt{\delta^{+}}$from the wall) within which the $V F$ term in (1) is of leading order, Wei et al. (2005); Klewicki, Ebner \& Wu (2011). Relative to the sketch of Fig. 2, this region encompasses layers I-III. Downstream of separation, the structure depicted in Fig. 2 erodes, and is eventually replaced by a new leading order balance that solely involves the $T I$ and $M I$ terms. These facts motivate the present section's examination of the Reynolds stress, and especially its profile evolution in the region where the $V F$ term loses its leading order magnitude.

For $x / \theta<10$ the blunt trailing edge $\langle u v\rangle^{+}$profiles of Fig. 9a exhibit expected boundary layer behaviors, except for $\eta$ values near to those that correspond to the splitter plate surface, i.e., for $|\eta|$ less than about 0.1 . Namely, when normalized using the $u_{\tau}$ estimate for the $10 \mathrm{~m} / \mathrm{s}$ boundary layer, the profiles respectively plateau at magnitudes near 1 and 0.5 on the high and low speed side. Close examination of the blunt trailing edge data further reveals that at $x / \theta=1.76$ and 2.94 the $\langle u v\rangle^{+}$profiles decrease from these boundary layer-like plateau values to near 0 at $\eta=-0.1$ and $\eta=0.1$, i.e., they continue to look very similar to the way they do in a boundary layer along a no-slip wall. Between $x / \theta \simeq 3$ and 6 , however, a distinctively sharp peak rapidly emerges. This part of the profile attains a maximal value more than twice that found in the boundary layer. In this context, it is also relevant to note that the peaks in the mean velocity gradient profiles (not shown) coincide closely with those in $\langle u v\rangle^{+}$for $x / \theta \geq 5$.88. Interestingly, however, the $d U^{+} / d \eta$ profile at $x / \theta=1.76$ also attains very large maxima, while, as just noted, the $\langle u v\rangle^{+}$profiles do not exhibit significant peaks until $x / \theta=5.88$. Collectively, these observations are taken to indicate that, relative to the sharp edge shear-wake, the onset of the instabilities of the separated shear-layers are slightly delayed in the blunt plate case.

The blunt trailing edge $\langle u v\rangle^{+}$profile at $x / \theta=11.76$ exhibits the same characteristic peaks as the profiles at $x / \theta=5.88$ and 8.82 , only now there is a continuous (nearly linear) variation in the region between the peaks. This is taken to be another indication that the two boundary layers begin to directly interact for $x / \theta$ greater than about 10 . With downstream evolution these profiles develop an increasingly dominant positive peak that is located on the high-speed side of $\eta=0$ (near $\eta=-0.12$ ) and centered within an increasingly broader distribution. The negative peak associated with the wake component on the low-speed side continually 

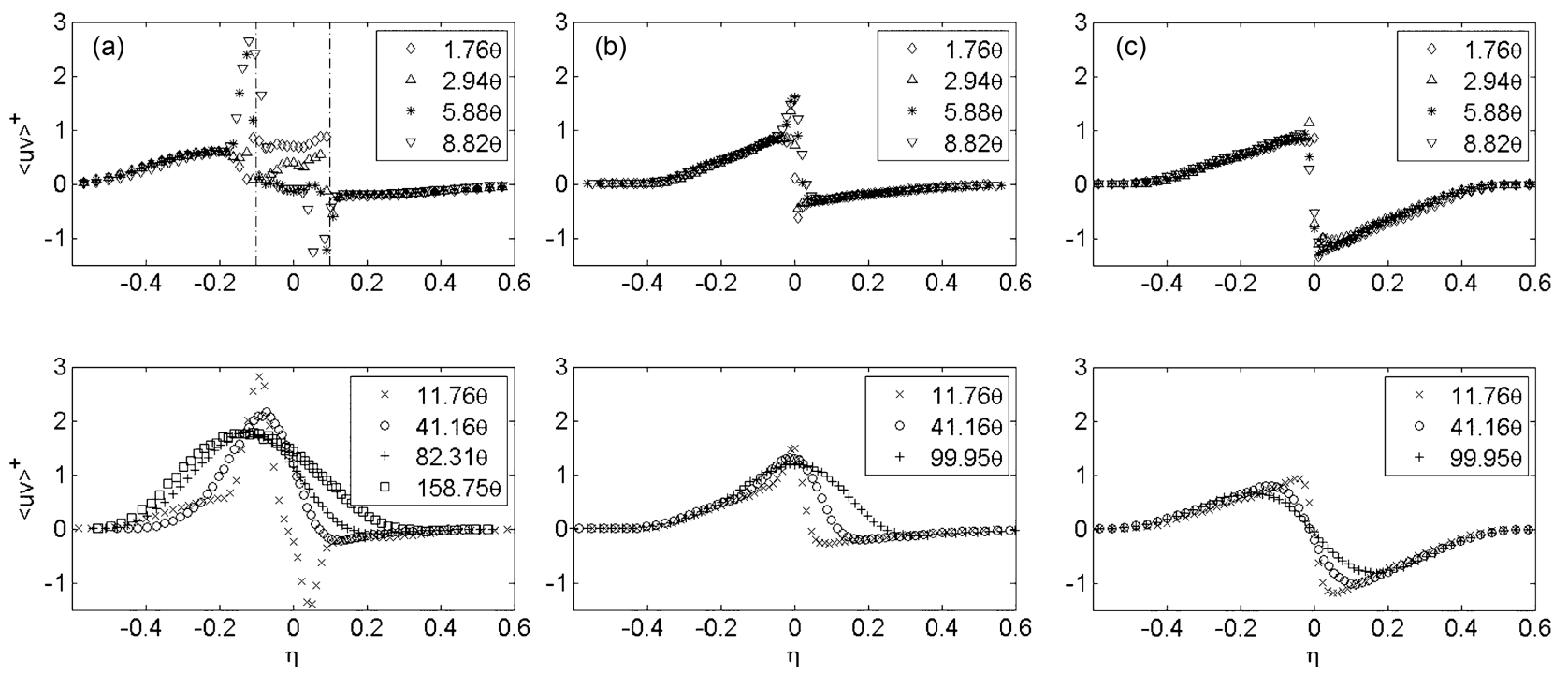

Fig. 9 Inner-normalized Reynolds stress profile evolution: (a) shear-wake flow downstream of the blunt trailing edge (dot-dash lines indicate the $\eta$ positions of the splitter plate surface), (b) shear-wake flow downstream of the sharp trailing edge, (c) wake flow downstream of the sharp trailing edge. Note that $\eta=y / \delta_{s w}$ and that entries in the legend denote streamwise location downstream of the splitter plate.

migrates to greater $\eta$ and diminishes in amplitude with increasing $x / \theta$.

The early development of the sharp trailing edge shear-wake (Fig. 9b) is marked by a pronounced peak in $\langle u v\rangle^{+}$that occurs in the shear layer caused by the abrupt end of the plate, and that significantly exceeds the peak value of the pre-separation boundary layer. The peak magnitude is not, however, as large as in the blunt plate case. Furthermore, unlike the blunt plate case, the profiles of Fig. 9b provide evidence that this peak develops before $x / \theta=5.88$. This is taken to result from the direct interaction between the wall-layer vorticity fields affiliated with the separating boundary layers, and thus this aspect is distinct from the blunt plate case. At locations farther downstream, the sharp and blunt trailing edge shear-wake $\langle u v\rangle^{+}$profiles exhibit similar behaviors, although the peak amplitudes at comparable $x / \theta$ remain smaller in the sharp edge case. Figure $9 \mathrm{~b}$ also reveals that with increasing $x / \theta$ the position of the dominant (positive) peak essentially centers on $\eta=0$. This observation gains consistency with the blunt trailing edge case if one correlates the position of this peak with the $\eta$ value where the highspeed flow separates.

For small $x / \theta$ the wake profiles in Fig. 9c provide evidence of a mildly increasing peak value in the vicinity of $\eta=0$. At $x / \theta=1.76$ the profile reaches a peak magnitude close to that found in the boundary layer, and then the profile apparently begins to turn toward zero. Moving inward from near one of the peaks, the profile then almost discontinuously transitions through zero to a value close to the other peak. Between $x / \theta=$ 1.76 and 8.82 the maximum amplitude of the profile increases only by about $15 \%$. With further increases in $x / \theta$, the peak values symmetrically attenuate, and move to greater $\eta$.

Independent of Reynolds number, the position, $y_{m}^{+}$, of the peak in the Reynolds stress (zero-crossing of the $T I$ term in (1)) is, by definition, centrally located within layer III of Fig. 2. Direct analysis of the mean momentum equation, along with a preponderance of empirical evidence, indicates that $y_{m}^{+}=\lambda \sqrt{\delta^{+}}$, with $\lambda \simeq 1.9$ Long \& Chen (1981); Afzal (1982); Sreenivasan \& Sahay (1997); Wei et al. (2005). In connection with this, Figs. 10a,b plot the outer- and innernormalized distances between the positive and negative $\langle u v\rangle^{+}$peaks as a function of $x / \theta$ and $x^{+}$, respectively. The data of Fig. 9c verify that wake profiles are symmetric about $\eta=0$ for all $x / \theta$, and that the distances between the $\langle u v\rangle^{+}$peaks are accurately given by twice the distance from $\eta=0$ to either peak. The downstream evolution of the $\langle u v\rangle^{+}$peak positions in this flow make for especially interesting comparison with those in the boundary layer. In the mean, the wake has a nopenetration condition at $\eta=0$, while the solid surface under the boundary layer instantaneously enforces both a no-penetration and no-slip condition.

For sufficiently large $x / \theta$, all of the present flows develop a region where the distance between the two $\langle u v\rangle^{+}$peaks increases at a rate $\sim x^{1 / 2}$. Under outernormalization (Fig. 10a), this region begins near $x / \theta=$ 10. Consistent with the existence of a logarithmic-like 

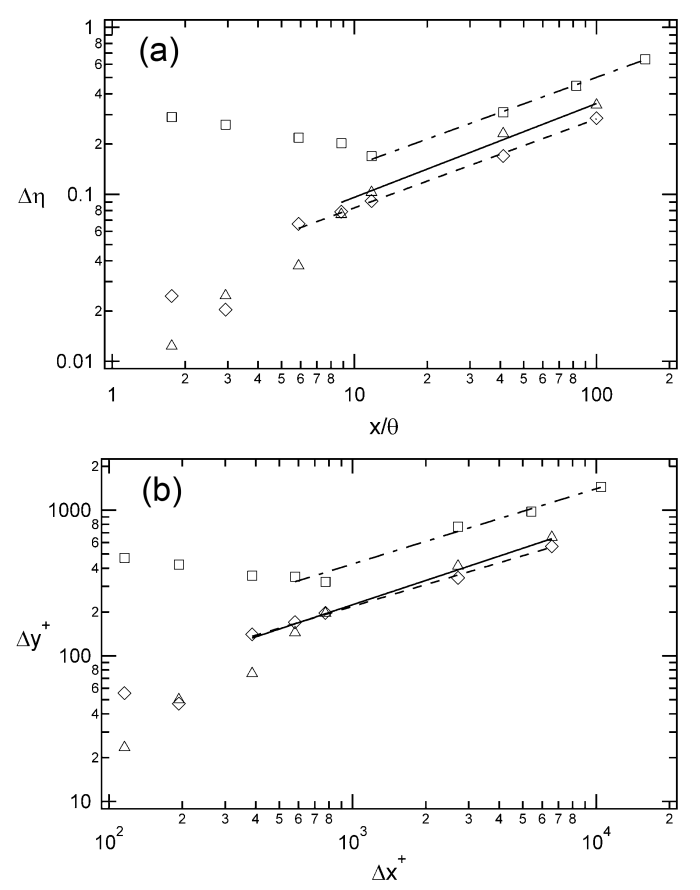

Fig. 10 Distance between the positive and negative peaks in the Reynolds stress profile: (a) Normalized by the shear layer width and plotted versus $x / \theta$, solid line, $\Delta \eta=$ $0.026(x / \theta)^{0.562}$, dashed line, $\Delta \eta=0.024(x / \theta)^{0.532}$, dot-dash line, $\Delta \eta=0.044(x / \theta)^{0.529}$. (b) Normalized by $\nu$ and $u_{\tau}$ and plotted versus $x^{+}$, solid line, $\Delta y^{+}=4.86\left(x^{+}\right)^{0.555}$, dashed line, $\Delta y^{+}=6.75\left(x^{+}\right)^{0.502}$, dot-dash line, $\Delta \eta=$ 12.03( $\left.x^{+}\right)^{0.517}$ Symbols same as in Fig. 4.

wake minimum profile (Fig. 6), under inner-normalization this region develops for $x^{+}$greater than about 300 . Comparison of the curve-fits in Figs. 10a and b suggests that the inner-normalized data are slightly more closely approximated by the square root dependence. Furthermore, under inner-normalization the sharp trailing edge shear-wake and wake data very nearly coincide. (Regarding the small differences between Figs. 10a and b note that while $\nu / u_{\tau}$ is a fixed quantity, $\delta_{s w}$ is a function of $x$.) For small streamwise distances $(x / \theta<10$, $x^{+}<300$ ), the sharp trailing edge data rise up to the $\sim x^{1 / 2}$ curve, and, although somewhat scattered, generally indicate that the shear-wake data reside above those from the wake. The blunt trailing edge data are distinct in this region, and are slightly decreasing prior to the region of $\sim x^{1 / 2}$ dependence.

While the inner-normalized profiles of Figs. 9a-c document the evolution of the Reynolds stress relative to an estimate of the wall shear stress at separation, an accounting of its evolution relative to the local state of the turbulence is given by the associated correlation coefficient, $\rho_{u v}=\langle u v\rangle /\left(u^{\prime} v^{\prime}\right)$, where the superscript prime denotes rms. These profiles are shown in Fig. 11. The $x / \theta=1.76$ blunt trailing edge profile of Fig. 11a further reinforces the data of Fig. 9a that, at this downstream location, the Reynolds stress profile outside of the $\eta$ position of the plate surface exhibits very little difference from the boundary layer profile - even in the region interior to $y_{m}$. Directly behind the plate, this $\rho_{u v}$ profile is positive (i.e., of the same sign as on the high speed side), and forms a nominally constant plateau, except near the plate edge on the low-speed side. Here it exhibits a positive peak that is immediately adjacent to a negative peak. These observations are interpreted to indicate that the initial behavior of the region directly behind the blunt plate is dominated by the influence of the flow on the high speed side. At larger $x / \theta$ locations, the positive plateau diminishes, and the profile evolves toward a smoother transition between the highspeed and low-speed streams. During this process, the positive and negative peaks in $\rho_{u v}$ diverge until they nominally align with positions just interior to the plate surfaces, see the profile at $x / \theta=11.76$. By $x / \theta=41.2$ the region behind the plate evolves to one of constant positive correlation, having a magnitude that is nominally the same as found in the boundary layer, i.e., $\simeq 0.45$. For $\eta$ values outside of the region directly behind the blunt plate, the $\rho_{u v}$ profile on either side of the plate is remarkably similar to that found in the boundary layer, and largely remains that way up to considerable distances downstream. Eventually, the negative plateau on the low-speed side begins to diminish as it moves to increasingly larger $\eta$, see profiles at $x / \theta=82.3$ and 158.8. This occurs in concert with the entire profile shifting toward low speed side.

A number of the features exhibited by the blunt trailing edge flow for $x / \theta$ positions downstream of where the boundary layers begin to interact $(x / \theta>10)$, are similarly exhibited by the sharp trailing edge flow. In this case, however, the corresponding similar interactions begin to occur at $x / \theta \simeq 0$. The closely juxtaposed positive and negative peaks for the smallest $x / \theta$ in the sharp edge shear-wake are qualitatively similar to those at $x / \theta=11.8$ in the blunt edge shear-wake, as are the profile behaviors for greater $x / \theta$ in each of these flows. One feature that is not present is the overall shift of the profile on high-speed side toward the low-speed side. This feature could, however, emerge at $x / \theta$ positions greater than explored in the present experiments. For $x / \theta<10$ in the sharp edge shear-wake, the deviation from the boundary layer $\rho_{u v}$ profile is highly localized around $\eta=0$, but for larger $x / \theta$ extends out to near $\eta=0.3$. In both shear-wake flows, the persistence of a significant negative peak on the low-speed side reveals that even by the farthest downstream locations the wake component still strongly influences the turbulence interactions, despite evidence of a highly attenu- 

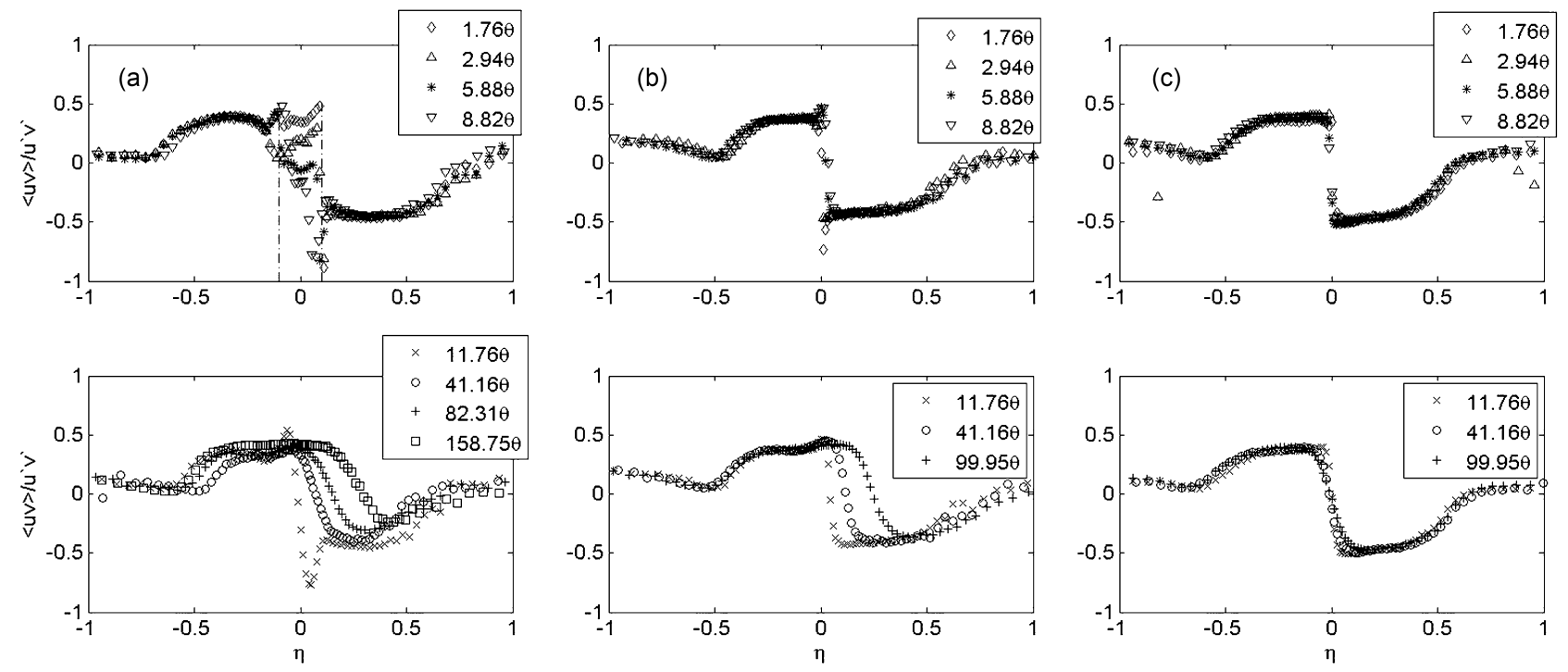

Fig. 11 Reynolds stress correlation coefficient profile evolution: (a) shear-wake flow downstream of the blunt trailing edge (dot-dash lines indicate the $\eta$ positions of the splitter plate surface), (b) shear-wake flow downstream of the sharp trailing edge, (c) wake flow downstream of the sharp trailing edge.

ated negative peak in the corresponding $\langle u v\rangle^{+}$profiles, and the fact that a wake deficit is barely visible in the mean profiles.

The $\rho_{u v}$ profiles for the wake (Fig. 11c) show little evidence of the positive and negative peaks exhibited near separation in the two shear-wake flows. Furthermore, for all $x / \theta$ investigated, the wake $\rho_{u v}$ profiles appear to exhibit (approximate) similarity under outer normalization, except in the narrow region centered on $\eta=0$, where $\rho_{u v}$ changes sign.

Comparison of Figs. 9 and 11 reveals that use of the turbulence intensities to normalize $\langle u v\rangle$ significantly attenuates the high amplitude peaks seen in the nearseparation profiles of $\langle u v\rangle^{+}$. Among other things, clarity regarding how and why this occurs is gained by examining the evolution of the inner-normalized streamwise and wall-normal velocity intensities. These are shown in Figs. 12 and 13 respectively. As can be seen, a number of the features affiliated with the $\langle u v\rangle^{+}$(and $\rho_{u v}$ ) profiles are also reflected in the $u^{\prime+}$, and especially the $v^{\prime+}$ profiles. For example, in both of the shear-wake flows the portion of the outer region where the differing $x / \theta$ profiles merge significantly diminishes at larger $x / \theta$.

Perhaps the most significant attributes of the profiles in Figs. 12 and 13 are revealed by considering their respective peak magnitudes relative the peak values found in the boundary layer. At these low Reynolds numbers, the peak amplitude of $u^{\prime+}$ is about 2.7 and this peak is located near $y^{+}=15$, e.g., Klewicki (2010). Examination of Fig. 12 thus reveals that the peak value in the sharp edge shear-wake only exceeds the expected boundary layer value by about $10 \%$, while in the wake the peak value never rises above the boundary layer value. In the blunt edge shear-wake a situation similar to that found in the sharp edge shear-wake is seen on the high speed side, while on the low-speed side there is a significantly increased magnitude relative to the expected boundary layer value. (Recall that the $u_{\tau}$ value from the high speed side is used to normalize the data on both sides of the shear-wake.) Consistently, this peak coincides with the strong positive $\langle u v\rangle$ peak near the $\eta$ position of the low-speed wall discussed relative to Fig. 11a. In contrast to the modest increases seen in $u^{\prime+}$, the shear-wake $v^{\prime+}$ data exhibit peaks well-above their boundary layer values. In the blunt shear-wake the $v^{\prime+}$ peaks exceed more than two times their boundary layer values. In the sharp edge shear-wake the observed peak increases by about $25 \%$, while in the wake, the increase is about $10 \%$. From these observations, it is thus seen that the post-separation peaks in $\rho_{u v}$ are attenuated relative to those observed in $\langle u v\rangle^{+}$predominantly owing to a nearly commensurate amplification of the wall-normal velocity intensities.

\subsection{Mean Momentum Balance}

One aim of the present experiments was to acquire profile measurements that were smooth enough to accurately estimate derivatives, and by doing so gain direct information regarding the evolution of the terms in (1). This objective is largely, but not wholly, attained. As noted in the discussion of Fig. 4, supplementary nearwall data were acquired to more accurately characterize 

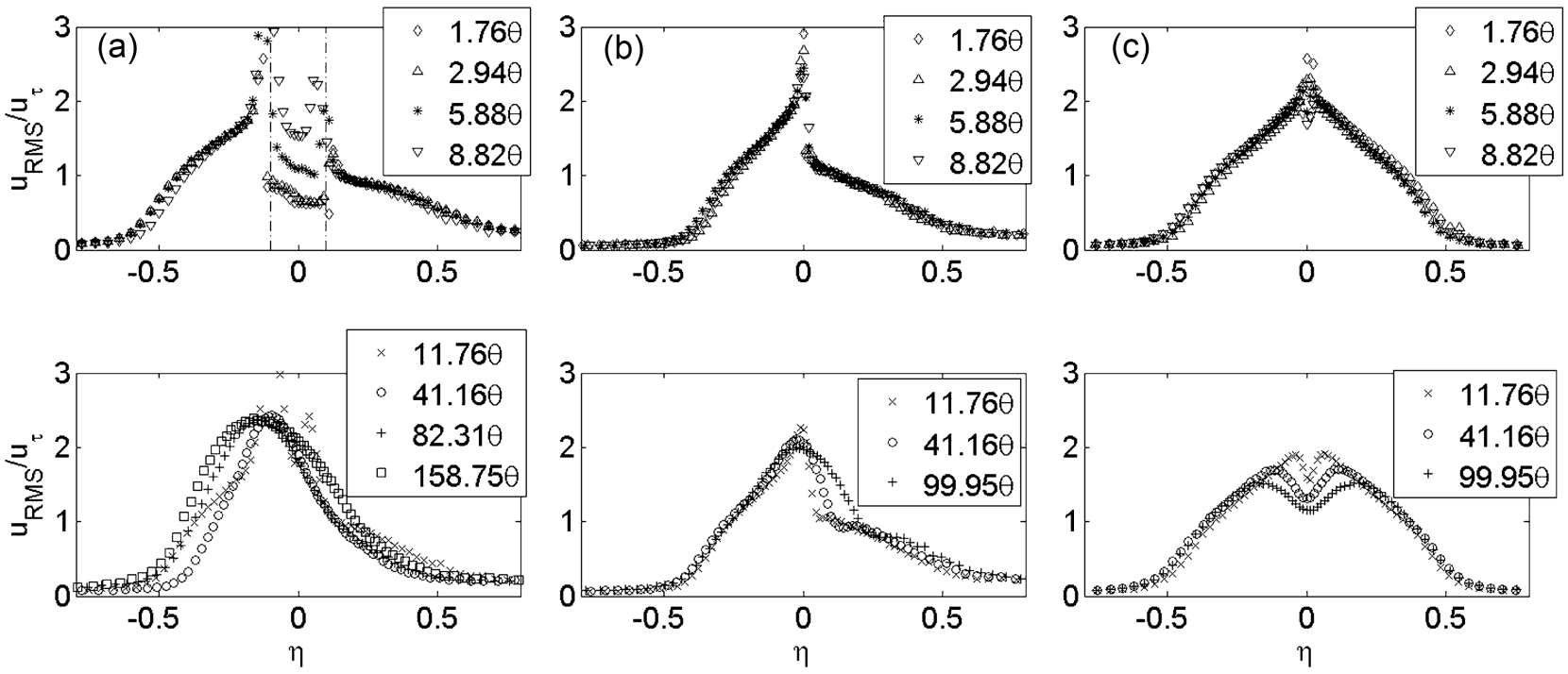

Fig. 12 Inner-normalized streamwise velocity intensity profile evolution: (a) shear-wake flow downstream of the blunt trailing edge (dot-dash lines indicate the $\eta$ positions of the splitter plate surface), (b) shear-wake flow downstream of the sharp trailing edge, (c) wake flow downstream of the sharp trailing edge.
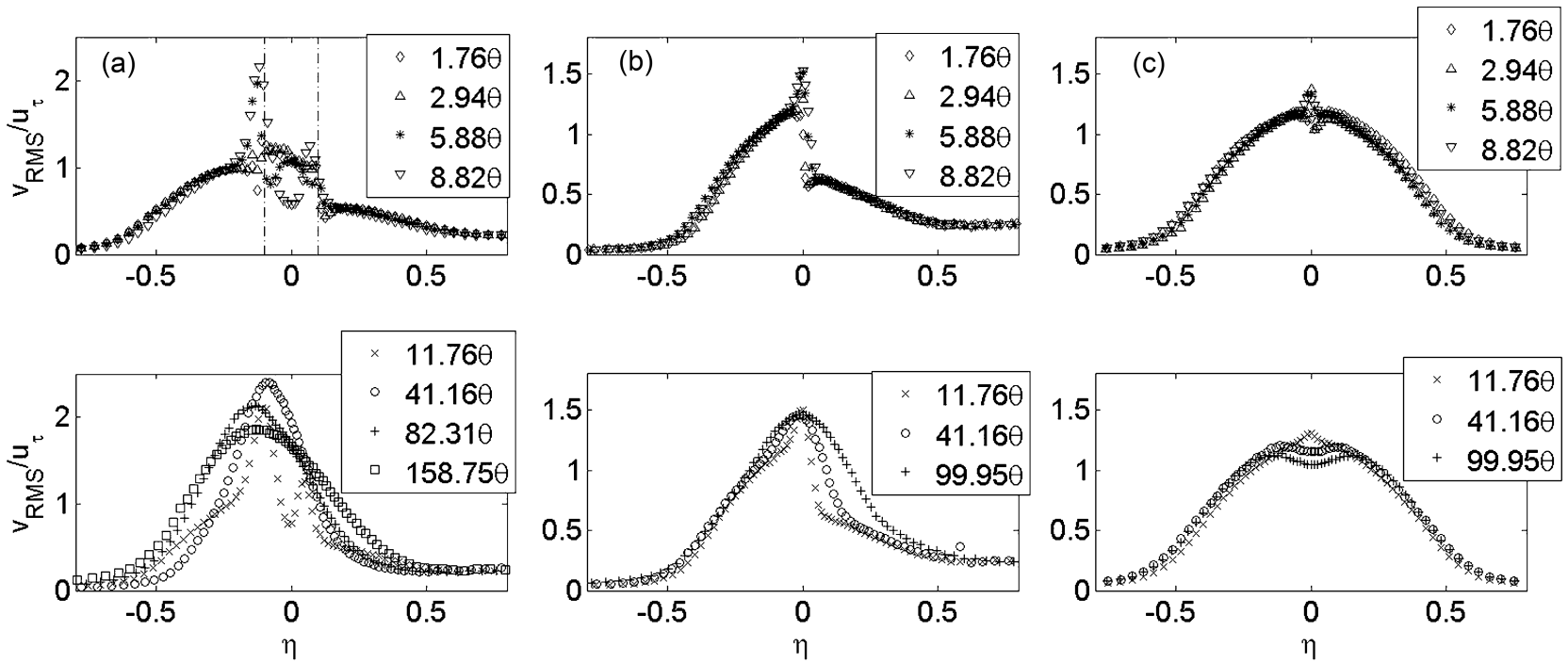

Fig. 13 Inner-normalized wall-normal velocity intensity profile evolution: (a) shear-wake flow downstream of the blunt trailing edge (dot-dash lines indicate the $\eta$ positions of the splitter plate surface), (b) shear-wake flow downstream of the sharp trailing edge, (c) wake flow downstream of the sharp trailing edge.

the near-wall profile shapes. The profiles in this section use these more finely spaced data. A number of the figures show, however, that the region immediately downstream of separation contains sharp mean gradients, that in some cases exhibit changes in sign over small $y$ increments. In the following we first explore the ratio of the $V F$ and $T I$ terms in (1) (see, Fig. 2), and then explore the relative influences of the $M I$ and streamwise gradient terms.

Figures 14 and 15 display inner-normalized profiles of the $V F$ and $T I$ terms at $x / \theta=1.76$ and 5.88 , as well as the profile of the ratio of these two quantities. In these figures the $y^{+}$origin of the sharp trailing edge experiments is set at the tip of the trailing edge, while for the blunt trailing edge experiment the $y^{+}=0$ position is at the corner of the blunt trailing edge. Plotting the data in this manner best facilitates an understanding of how the boundary layer structure erodes downstream of separation. The $V F$ and $T I$ profiles from all of the experiments exhibit smooth and discernible trends. It is apparent, however, that for small $y^{+}$the profiles ex- 
hibit much more variation per spatial increment than for larger $y^{+}$.

The third graph in each panel reveals that there is considerable scatter in the profile of $V F / T I$. For larger $y^{+}$distances, this scatter is reliably attributable to taking the difference of two small numbers. Thus, this is generally taken to underlie the identifiably non-zero values of $V F / T I$ for $y^{+}$greater than about 80 . Nearer to $y^{+}=0$, the $V F$ and $T I$ profiles have larger magnitudes, and thus, in the absence of other effects, the signal-tonoise ratio is expected to decrease. Relatively small errors in the ability to capture the large amplitude and rapidly varying profiles at small $y^{+}$apparently, however, introduces uncertainties.
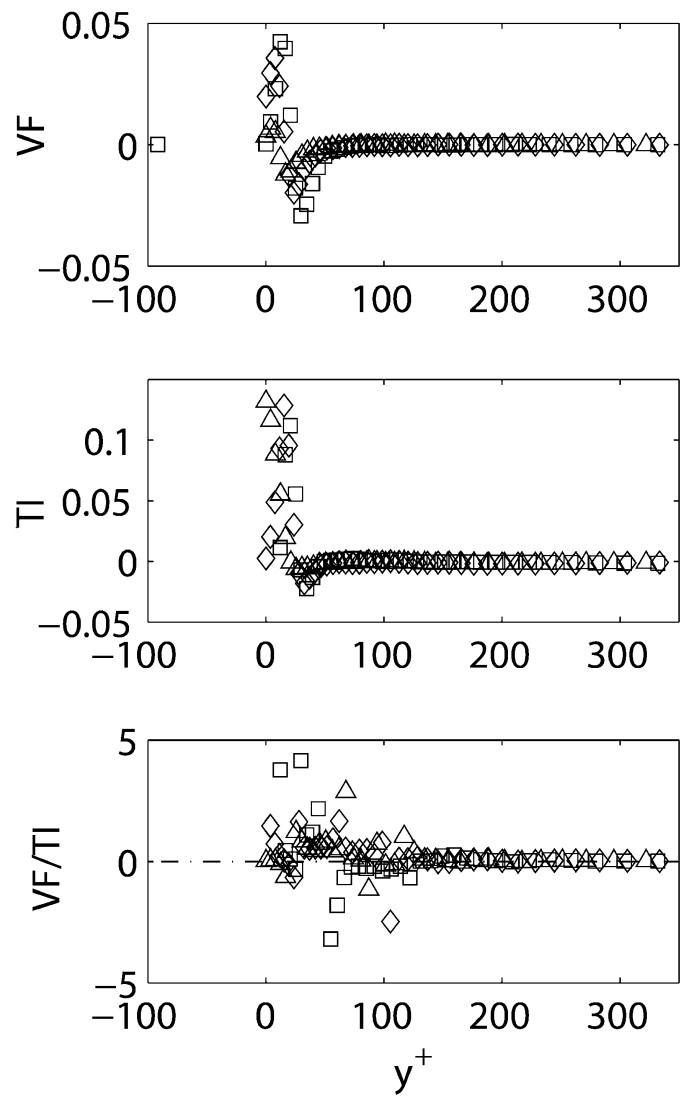

Fig. 14 Profiles of the mean viscous force $(V F)$, the mean effect of turbulent inertia $(T I)$, and their ratio at $x / \theta=1.76$ in the $10 \mathrm{~m} / \mathrm{s}$ stream. Symbols same as in Fig. 4.

From the upper two plots of Figs. 14 and 15 it is apparent that the near-wall flow evolution immediately downstream of separation is simultaneously marked by attenuation of the $V F$ term and amplification of the $T I$ term. Canonical boundary layer data indicate that the inner-normalized $V F$ profile is strictly negative, attains a near-wall peak of about -0.07 , and is identically zero at the wall. The replacement of the no-slip con-
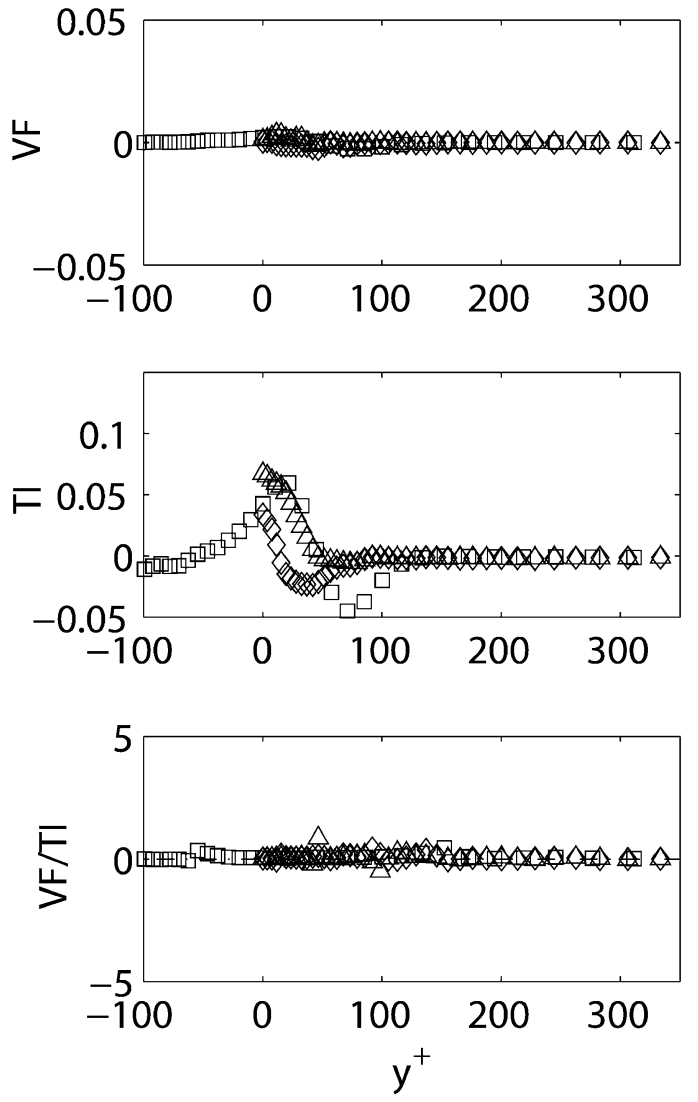

Fig. 15 Profiles of the mean viscous force $(V F)$, the mean effect of turbulent inertia $(T I)$, and their ratio at $x / \theta=5.88$ in the $10 \mathrm{~m} / \mathrm{s}$ stream. Symbols same as in Fig. 4.

dition with a non-zero and increasing wake minimum results in a rapid attenuation in this profile's negative peak, and underlies the generation of a distinct positive peak nearer to $y^{+}=0$. This positive peak is at first most prevalent in the blunt shear-wake profile. Between $x / \theta=1.76$ and $x / \theta=5.88$, however, both the positive and negative $V F$ peaks in the blunt shear-wake essentially disappear.

Given the sources of uncertainty discussed above, only limited conclusions from the $T I / V F$ profiles of Figs. 14 and 15 are warranted. It seems clear, however, that by $x / \theta \simeq 6$ the flow interior to $y_{m}^{+}$becomes dominated by the inertial terms in (1). We now seek to better understand how this occurs by examining the estimated behaviors of the streamwise gradient and $M I$ terms in (1). The $V \partial U / \partial y$ profiles were calculated by finite differencing the $U$ profiles at $x / \theta=1.76,2.94$ and 5.88, and multiplying by the measured $V$ values at those locations, respectively. The $U \partial U / \partial x$ and $\partial\left(\left\langle u^{2}\right\rangle-\left\langle v^{2}\right\rangle\right) / \partial x$ terms required differencing of profiles measured at different streamwise locations. Thus, the positions of these profiles $(x / \theta=2.35,4.41$ and 7.35$)$ are offset from the others. This fact, coupled with the rapid streamwise 

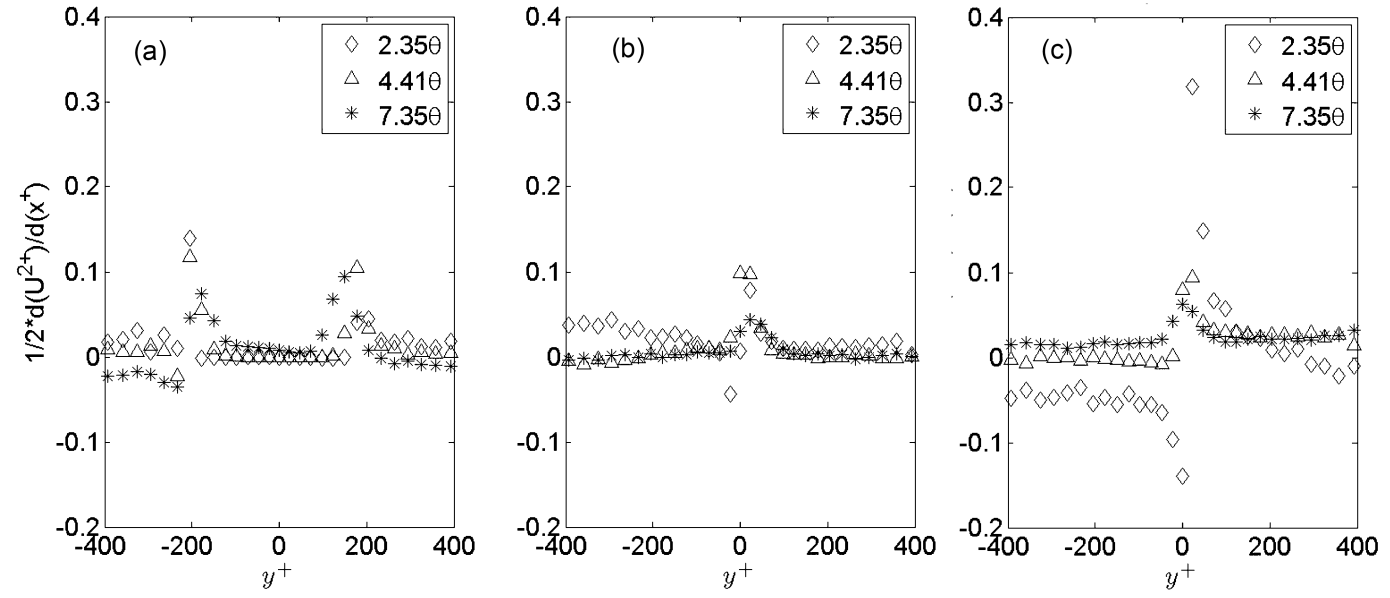

Fig. 16 Estimates of the inner-normalized profiles of $U \partial U / \partial x$ downstream of separation (a) blunt trailing edge shear-wake, (b) sharp trailing edge shear-wake, (c) sharp trailing edge wake.
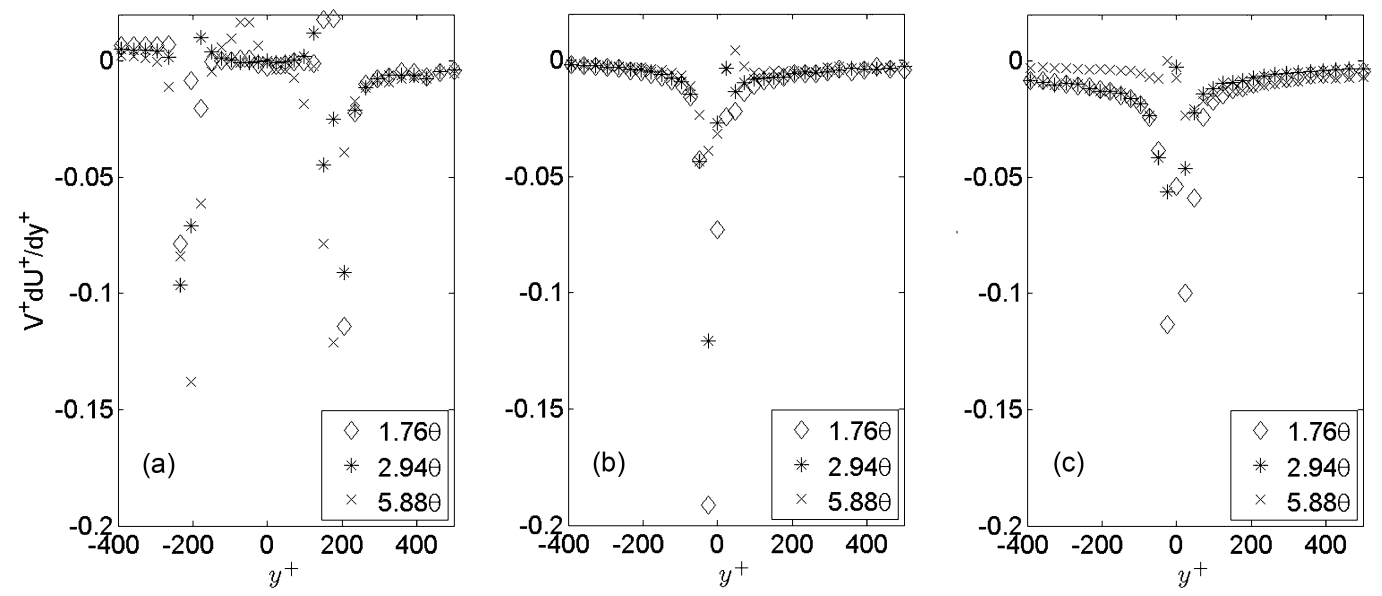

Fig. 17 Estimates of the inner-normalized profiles of $V \partial U / \partial y$ downstream of separation (a) blunt trailing edge shear-wake, (b) sharp trailing edge shear-wake, (c) sharp trailing edge wake.
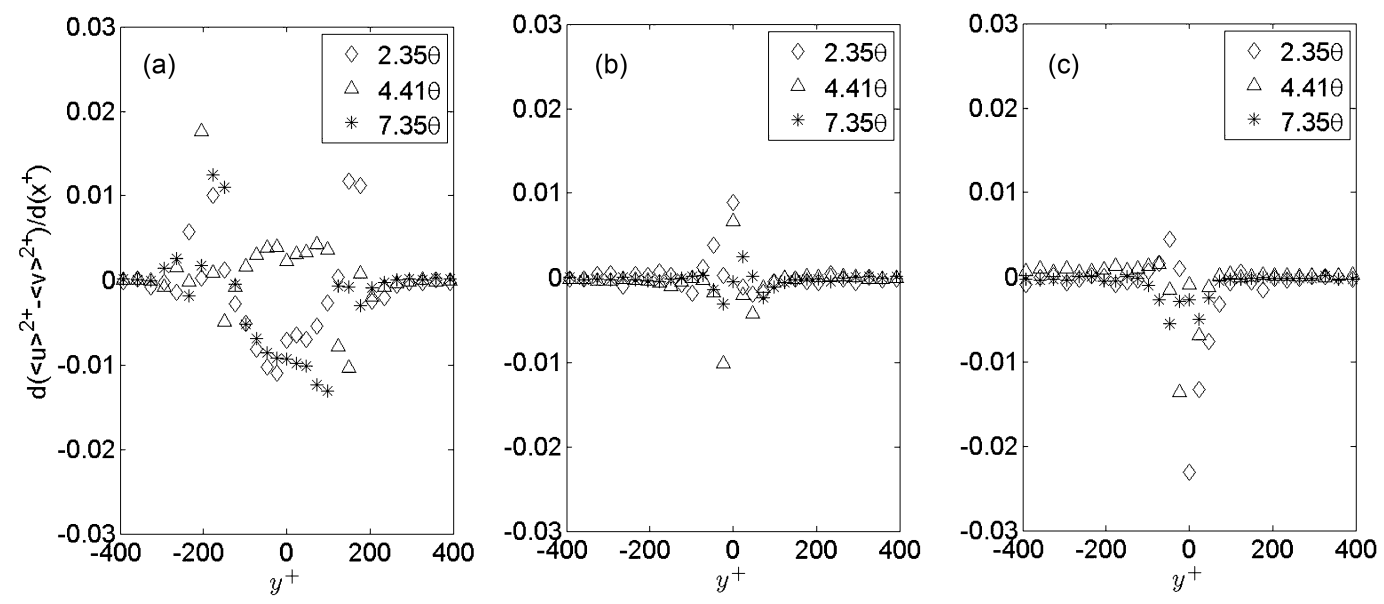

Fig. 18 Estimates of the inner-normalized profiles of $\partial\left(\left\langle u^{2}\right\rangle-\left\langle v^{2}\right\rangle\right) / \partial x$ downstream of separation (a) blunt trailing edge shear-wake, (b) sharp trailing edge shear-wake, (c) sharp trailing edge wake. 
flow development in the region $x / \theta \leq 10$, precludes a precise reconstruction the mean momentum balance at any given $x / \theta$ value. An estimate of the uncertainties that includes this offset effect is obtained by examining the residual of the terms in (1) using the profile data at $x / \theta=5.88$ or 7.35 . When this is done, the magnitude of the residual is consistently found to be about the size of the smallest non-zero term. From such considerations it is thus concluded that the profiles in Figs. 14 - 18 accurately reflect the relative magnitudes of the terms, but owing to the offset effect the profiles at even slightly different $x / \theta$ cannot be used to reconstruct the entire balance of (1).

Inner-normalized profiles of the $U \partial U / \partial x, V \partial U / \partial y$, and $\partial\left(\left\langle u^{2}\right\rangle-\left\langle v^{2}\right\rangle\right) / \partial x$ terms are shown in Figs. $16-$ 18, respectively. As expected, the $U \partial U / \partial x$ profiles are positive and $V \partial U / \partial y$ profiles are negative. (Note that the negative excursion in the $x / \theta=2.35$ wake profile is believed to be anomalous.) Physically, these behaviors are affiliated with the rapid influx of mean momentum into the region of wake deficit. At any given $x / \theta$, the $V \partial U / \partial y$ term generally has larger magnitude than the $U \partial U / \partial x$ term, and thus this is consistent with a positive TI term largely accounting for the net balance of (1). The data of Fig. 18 indicate that in the vicinity of separation the $\partial\left(\left\langle u^{2}\right\rangle-\left\langle v^{2}\right\rangle\right) / \partial x$ term is smaller, but not negligible relative to the other inertial terms. Consistent with the more significant relative changes in the $v^{\prime+}$ profiles noted previously, the largest excursions in $\partial\left(\left\langle u^{2}\right\rangle-\left\langle v^{2}\right\rangle\right) / \partial x$ tend to be negative. Overall, the data of Figs. 16 - 18 suggest that by $x / \theta \simeq 8$, the $M I$ and $T I$ terms comprise the two leading order terms in (1). In terms of the inflow boundary layer scales, the length of this region is $x \simeq \delta$, where $\delta$ is the boundary layer thickness at separation. Thus, it is within this region that the flow transitions from being well-represented by the canonical boundary layer equation, $M I=V F+T I$, to being well-represented by the turbulent wake equation $M I=T I$.

According to the analysis of Bogucz \& Walker (1988) there is a much smaller fetch, starting just prior to separation and ending just after separation, within which the boundary layer equations lose validity. Based upon classical asymptotic estimates, they determine that the overall length of this region is $O\left(\nu / u_{\tau}\right)$, or more precisely, of the order of the thickness of the $y$ direction sub-domain upon which $V F=T I$ is the leading order approximation to the mean momentum equation. Their estimate for the thickness of this region is, however, not correct. The region where $V F=T I$ is precisely the thickness of layer II in Fig. 2, which has been analytically determined and empirically verified to be $O\left(\sqrt{\nu \delta / u_{\tau}}\right)$, e.g., Wei et al. (2005); Fife et al. (2009).
Given the low $\delta^{+}$of the present experiments, it is doubtful that the first measurement station is within this domain. Alternatively, to within their accuracy the present measurements support the validity of the thin shear layer approximation of (1) at each $x / \theta$ location.

The above results clarify how the influence of the $V F$ term in (1) loses its leading order importance in the region $y<y_{m}$ for $x / \theta<8$. For greater $x / \theta$ values, the flow development is therefore effectively governed by an inertially dominated mean force balance for both $y<y_{m}$ and $y>y_{m}$. Primary characteristics of the flow development outside of the near-separation region include a variation in the minimum wake velocity like $\ln \left(x^{+}\right)$(Fig. 6), and $\langle u v\rangle^{+}$peak positions that move apart like $\sqrt{x^{+}}$(Fig. 10). According to the wake formulation of Bogucz \& Walker (1988), the logarithmic dependence of $U_{m}^{+}$on $x^{+}$is affiliated with the $T I$ term in (1) decreasing like $1 / x$ along the wake center. (Note that the TI term attains its maximum value at the wake center.) In connection with this, Fig. 19 plots the maximum value of $|\partial\langle u v\rangle / \partial \eta|$ as a function of $x / \theta$. For $x / \theta \geq 5.88$ the data in this figure closely follow the indicated power-law dependence. Somewhat remarkably, this dependence appears to hold regardless of the trailing edge geometry or the presence of overall shear. In comparison with the theory of Bogucz \& Walker (1988), the present $|\partial\langle u v\rangle / \partial \eta|_{\text {max }}$ data apparently decrease slightly more slowly, as they are best fit by a curve that varies like $x^{-0.8}$, as opposed to $x^{-1}$.

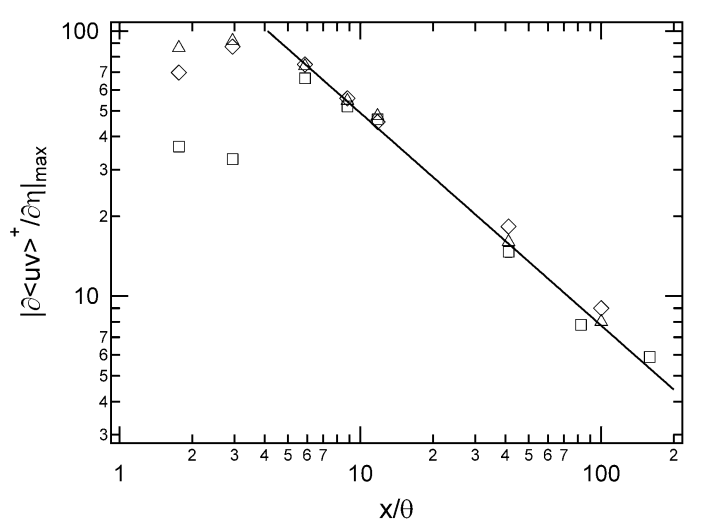

Fig. 19 Maximum magnitude of $\partial\langle u v\rangle / \partial \eta$ plotted versus $x / \theta$. Solid line is given by $|\partial\langle u v\rangle / \partial \eta|_{\max }=311(x / \theta){ }^{-0.802}$ Symbols same as in Fig. 4.

\section{Conclusions}

The results from the present experiments provide a basis for understanding the factors influencing the downstream development of shear-wake flows that originate 
from turbulent boundary layers. These factors include, the Reynolds numbers of the boundary layers at separation, the effect of the overall shear (relative to the pure wake flow), as well as plate geometry effects that include the trailing edge geometry and the pressure gradient caused by the plate taper. Below we present a relatively brief synopsis of what we have learned.

The wake studies reviewed in the Introduction indicate that for a significant distance downstream of separation the flow field evolution continues to be characterized by the viscous scales of the boundary layer at separation. The data of Figs. 6 and 19 support this general conclusion, and extend it to indicate that the influence of a mean shear has little qualitative effect; albeit one has to account for the fact that the wake minimum exhibits an approximately linear shift toward the high speed flow with increasing downstream development. To the author's knowledge, the data of Fig. 10 are the first to reveal that the wall-normal distance between the peaks in the Reynolds stress in this region move apart like $\sqrt{x}$. This is reminiscent of what occurs in the boundary layer where $y_{m}^{+} \sim \sqrt{\delta^{+}}$. Remarkably, the present results indicate that all of these features are also established for shear-wakes whose boundary layers at first do not directly interact downstream of separation, i.e., as in the blunt trailing edge flow. It is rational, however, to expect that this conclusion will no longer hold when the plate thickness becomes large.

The data documenting the downstream development of the Reynolds stress and turbulence intensities provide evidence that, for the sharp trailing edge cases, the adverse pressure gradient affiliated with the taper of the splitter plate has a noticeable effect. Under all cases, however, the shear layer interactions immediately downstream of separation cause an abrupt intensification of the turbulence intensities. This effect is most dramatically revealed in the blunt shear-wake, and to a decreasing extent in the sharp shear-wake and wake, respectively. The shear layer interactions immediately downstream of separation also lead to distinctive peaks in the Reynolds stress. Examination of the Reynolds stress correlation coefficient reveals that these peaks correlate with a disproportionate amplification of the wall-normal velocity intensities. In the outer region, and at farther downstream locations, the profiles of the turbulence intensities and Reynolds stress approximately scale on the outer length scale in the sharp trailing edge flows, but undergo a more noticeable shift toward the high speed side in the blunt trailing edge shear-wake.

Estimates of the terms in the mean momentum equation reveal the existence of two regions of streamwise development. The approximate region $0<x / \theta<8$ is characterized by the rapid attenuation of the $V F$ term relative to the other terms in (1). In this region, the $y<y_{m}$ portion of the separated boundary layer is shown to become dominated by inertial mechanisms. This occurs owing to an amplification of the TI term, a reduction in the $V F$ term, and leading order contributions from the streamwise gradient and $M I$ terms. For $x / \theta>8$, the leading order balance rapidly becomes one that solely involves the TI and MI terms. Consideration of these observations in concert with those pertaining to the development of the mean flow suggests that a logarithmic $U_{m}^{+}\left(x^{+}\right)$profile does not develop until the center of the wake component is, in the mean, dominated by inertial mechanisms. In this regard, the boundary layer structure depicted in Fig. 2 indicates that the thickness of the region where the $V F$ term is dominant in (1) scales like $\sqrt{\delta^{+}}$. Furthermore, both measurements and analytical estimates indicate that the velocity increment across this region is close to $0.5 U_{\infty}$ independent of $\delta^{+}$(Wei et al. , 2005). Thus, at high Reynolds number this region constitutes a diminishing fraction of $\delta$, while simultaneously possessing an inner-normalized thickness that is continually increasing - even though its mean circulation (per unit length) is a fixed fraction of $U_{\infty}$. This leads one to suspect that the streamwise distance over which the $V F$ term loses leading order importance is, in general, Reynolds number dependent. These considerations also support the conjecture that the vorticity enclosed within the region where $V F$ is of dominant order corresponds to the sub-shear layer as described by Morris \& Foss (2003). Lastly, since an adverse pressure gradient is associated with a surface flux of opposing sign mean vorticity, this effect will attenuate the near-wall curvature of $U^{+}\left(y^{+}\right)$ prior to separation. Thus, the adverse pressure gradient resulting from the splitter plate taper is likely to hasten the development of a momentum balance with inertial leading order terms.

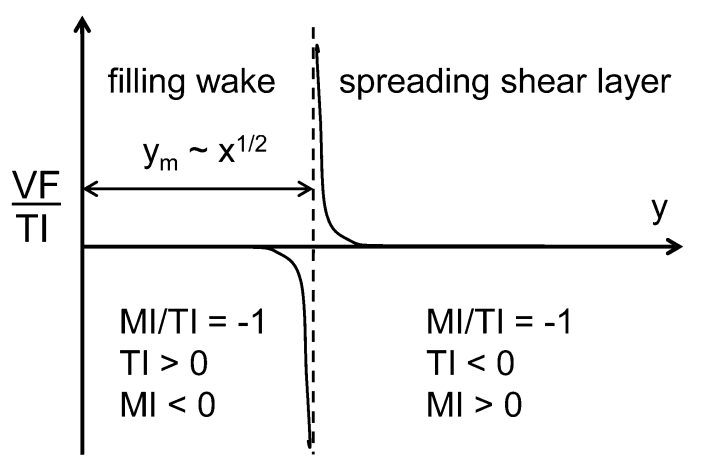

Fig. 20 Sketch of the ratio of $V F$ to $T I$ in the developing shear-wake for $x / \theta>8$. 
Once in the region where $T I$ and $M I$ are the sole leading order terms, the balance expressed by (1) is graphically depicted by Fig. 20. In the wake, this depiction remains valid until all of the mean vorticity is annihilated. In the shear-wake it is retained until there is only one sign of $\Omega_{z}$, i.e., when the shear-wake becomes a mixing layer. Like in the boundary layer, the $T I$ term passes through zero on either side of $\eta=0$ (here only shown on one side). Also, it is relevant to recall that $T I=0$ corresponds to a peak in the Reynolds stress profile. In the boundary layer the $T I$ zero-crossing is associated with a balance breaking and exchange of leading order terms in (1) such that for positions closer to the wall the balance is essentially between $V F$ and $T I$, and for greater wall-normal positions the balance is essentially between $T I$ and $M I$. In contrast, the $T I$ zerocrossing in the shear-wake corresponds to a commensurate change in the sign of $M I$. At present, the width of the region (surrounding the zero-crossing) where the $V F$ term might regain leading order is unknown, although the data of Fig. 15 would seem to indicate that it is very narrow. To within the capacity of the present measurements to detect a peak in the Reynolds stress one can, however, be assured that the zero-crossing exists. As depicted in Fig. 20, ramifications of the TI zerocrossing are that near the center of the wake component the mean effect of turbulent inertia drives an influx of mean momentum, and outside of this region this net effect of the turbulence drives the outflux of mean momentum responsible for the downstream spread of the flow.

Acknowledgements The authors are pleased to acknowledge the efforts of Caleb Morrill-Winter and Rachel Ebner in developing the hotwire sensors employed in the present study. This work was partially supported by the Office of Naval Research, grant no. N000140810836, Ronald Joslin grant monitor.

\section{References}

Afzal, N. (1982) Fully developed turbulent flow in a pipe: An intermediate layer. Ing.-Arch. 52: 355-377.

Alber, I.E. (1980) The turbulent wake of a thin flat plate. AIAA J. 18: 1044-1051.

Andreoploulos, J. and Bradshaw, P. (1980) Measurements of interacting turbulent shear layers in the near wake of a flat plate. J. Fluid Mech. 100: 639-668.

Bamberger, M. (2011) On the Downstream Evolution of the Mean Momentum Field in Turbulent Shear-Wake Flows. M.S. Thesis, University of New Hampshire.
Bogucz, E.A. and Walker, J.D.A. (1980) The turbulent near wake at a sharp trailing edge. J. Fluid Mech. 196: $555-584$.

Challa, D. (2005) On the Downstream Evolution of Turbulent Initial Condition Shear-Wake Flows. M.S. Thesis, University of Utah.

Chevray, R. and Kovasznay, L.S.G. (1969) Turbulence measurements in the wake of a thin flat plate. $A I A A$ J. 7: 1641-1643.

Dahm, W.J.A., Frieler, C.E., and Tryggvasan G. (1992) Vortex structure and dynamics in the near field of a coaxial jet. J. Fluid Mech. 241: 371-402.

Fife, P., Wei, T., Klewicki, J. and McMurtry, P. (2005) Stress gradient balance layers and scale hierarchies in wall bounded turbulent flows. J. Fluid Mech. 532: 165-189.

Fife, P., Klewicki, J. and Wei, T. (2009) Time averaging in turbulence settings may reveal an infinite hierarchy of length scales. J. of Discrete and Continuous Dynamical Systems 24: 781-807.

Folz, A. and Wallace, J.M. (2009) Near-surface turbulence in the atmospheric boundary layer. Physica $D$ 239: 1305-1317.

Haji-Haidari, A. and Smith, C.R. (1988) The development of the turbulent near wake of a tapered thick plate. J. Fluid Mech. 189: 135-163.

Klewicki, J. (2010) Reynolds number dependence, scaling and dynamics of turbulent boundary layers. $J$. Fluids Eng. 132: 094001.

Klewicki, J., Ebner, R. and Wu, X. (2011) Mean dynamics of transitional boundary-layer flow. J. Fluid Mech. 682: 617-651.

Klewicki, J. and Falco, R. (1990) On accurately measuring statistics associated with small-scale structure in turbulent boundary layers using hot-wire probes. J. Fluid Mech. 219: 119-142.

Ko, M.W.M. and Lam, K.M. (1989) Flow structure of coaxial jet of mean velocity ratio of 0.5 . AIAA J. 27: 513-514.

Koochesfahani, M.M. and Frieler, C.E. (1989) Instability of nonuniform density free shear layers with a wake profile. AIAA J. 27: 1735-1740.

Long, R. and Chen, T.-C (1981) Experimental evidence for the existence of the mesolayer in turbulent systems. J. Fluid Mech. 105: 19-59.

Mehta, R. (1991) Effect of velocity ratio on plane mixing layer development: Influence of the splitter plate wake. Expts. in Fluids 10: 194-204.

Morris, S. and Foss, J. (2003) Turbulent boundary layer to single-stream shear layer: The transition region. $J$. Fluid Mech. 494: 187-221.

Nakayama, A. and Liu, B. (1990) The turbulent near wake of a flat plate at low Reynolds number. J. Fluid 
Mech. 217: 93-114.

Sadr, R. and Klewicki, J.C. (2003) An experimental investigation of the near field flow development in coaxial jets. Phys. Fluids 15: 1233-1246.

Schlatter, P. and Orlu, R. (2010) Assessment of direct numerical simulation data of turbulent boundary layers. J. Fluid Mech. 659: 116-126.

Sreenivasan, K.R. and Sahay, A. (1997) The persistence of viscous effects in the overlap region and the mean velocity in turbulent pipe and channel flows. In Self-Sustaining Mechanisms of Wall Turbulence (Ed. R. Panton) Computational Mechanics Publications, Southampton, 253-272.

Vukoslavcevic, P., Wallace, J.M., and Balint, J.-L. (1991) The velocity and vorticity vector fields of a turbulent boundary Layer, Part I. Simultaneous measurement by hot-wire anemometry. J. Fluid Mech. 228: 25-51.

Vukoslavcevic, P. and Wallace, J.M. (1996) A twelvesensor hot wire probe to measure the velocity and vorticity vectors in turbulent flow. Meas. Sci. and Tech. 10: 1451-1461.

Wait, J. (2003) On the Downstream Evolution of Laminar Initial Condition Shear-Wake Flows. M.S. Thesis, University of Utah.

Wei, T., Fife, P., Klewicki, J. \& McMurtry, P. (2005) Properties of the mean momentum balance in turbulent boundary layer, pipe and channel flows. J. Fluid Mech. 522: 303-327.

Wei, T., Fife, P. and Klewicki, J. (2007) On scaling the mean momentum balance and its solutions in turbulent Couette-Poiseuille flow. J. Fluid Mech. 573: 371-398. 


\section{University Library}

\section{- M M N E R VA A gateway to Melbourne's research publications}

Minerva Access is the Institutional Repository of The University of Melbourne

Author/s:

Bamberger, M;Klewicki, J

Title:

Statistical structure and mean dynamics of developing turbulent shear-wake flows

Date:

2013-01-01

Citation:

Bamberger, M. \& Klewicki, J. (2013). Statistical structure and mean dynamics of developing turbulent shear-wake flows. EXPERIMENTS IN FLUIDS, 54 (1), https://doi.org/10.1007/ s00348-012-1415-0.

Persistent Link:

http://hdl.handle.net/11343/282686 